\title{
Direct numerical simulations of dense suspensions: wave instabilities in liquid-fluidized beds
}

\author{
J. J. DERKSEN ${ }^{1}$ AND S. SUNDARESA N ${ }^{2}$ \\ ${ }^{1}$ Multi-Scale Physics Department, Delft University of Technology, Prins Bernhardlaan 6, \\ 2628 BW Delft, The Netherlands \\ j.j.derksen@tudelft.nl \\ ${ }^{2}$ Department of Chemical Engineering, Princeton University, Princeton, NJ 08544, USA
}

(Received 19 May 2006 and in revised form 26 April 2007)

We present results of direct numerical simulations of travelling waves in dense assemblies of monodisperse spherical particles fluidized by a liquid. The cases we study have been derived from the experimental work of others. In these simulations, the flow of interstitial fluid is solved by the lattice-Boltzmann method (LBM) and the particles move under the influence of gravity, hydrodynamic forces stemming from the LBM, subgrid-scale lubrication forces and hard-sphere collisions. We first show that the propagating inhomogeneous structures seen in the simulations are in agreement with those observed experimentally. We then use the detailed information contained in the simulation results to assess aspects of two-fluid model closures, namely, fluid-particle drag, and the various contributions to the effective stresses. We show that the rates of compaction and dilation of the particle phase in the travelling waves are comparable to the rate at which the microstructure relaxes, and that there is a pronounced effect of the rate of compaction on the average collisional normal stress. Although this effect can be expressed as an effective bulk viscosity term, this approach would require the use of a path-dependent bulk viscosity. We also find that the effective fluid-particle drag coefficient can be described well with the often-used closure motivated by the experiments of Richardson \& Zaki (Trans. Inst. Chem. Engng vol. 32, 1954, p. 35). In this respect, the effect of the system size for determining the drag requires specific care. The shear viscosity of the particle phase manifests small, but clearly noticeable dependence on the rate of compaction/dilation of the particle phase. Our observations point to the need for higher-order closures that recognize the slow evolution of the microstructure in these flows and account for the effects of non-equilibrium microstructure on the stresses.

\section{Introduction}

Dense fluidized beds exhibit a rich variety of complex inhomogeneous flow structures, ranging from one-dimensional travelling waves to bubble-like voids. The origin and hierarchy of these structures in liquid-fluidized beds have been the subject of many theoretical and experimental studies (Anderson \& Jackson 1969; El-Kaissy \& Homsy 1976; Didwania \& Homsy 1981; Batchelor 1988; Ham et al. 1990; Anderson, Sundaresan \& Jackson 1995; Poletto, Bai \& Joseph 1995; Singh \& Joseph 1995; Glasser, Kevrekidis \& Sundaresan 1996, 1997; Zenit, Hunt \& Brennan 1997; Zenit \& Hunt 2000; Duru \& Guazzelli 2002; Duru et al. 2002). Most of the theoretical 
and computational studies probing these structures are based on the analysis of Eulerian two-phase-flow model equations, which treat the fluid and particle phases as interpenetrating continua. These two-fluid model equations take the following form:

$$
\begin{gathered}
\frac{\partial\left(\rho_{s} \phi\right)}{\partial t}+\nabla \cdot\left(\rho_{s} \phi \boldsymbol{v}\right)=0, \\
\frac{\partial\left(\rho_{f}(1-\phi)\right)}{\partial \mathrm{t}}+\nabla \cdot\left[\rho_{f}(1-\phi) \boldsymbol{u}\right]=0, \\
{\left[\frac{\partial\left(\rho_{s} \phi \boldsymbol{v}\right)}{\partial t}+\nabla \cdot\left(\rho_{s} \phi \boldsymbol{v} \boldsymbol{v}\right)\right]=\nabla \cdot \boldsymbol{\sigma}_{s}+\boldsymbol{F}+\rho_{s} \phi \boldsymbol{g},} \\
{\left[\frac{\partial\left(\rho_{f}(1-\phi) \boldsymbol{u}\right)}{\partial \mathrm{t}}+\nabla \cdot\left(\rho_{f}(1-\phi) \boldsymbol{u} \boldsymbol{u}\right)\right]=\nabla \cdot \boldsymbol{\sigma}_{f}-\boldsymbol{F}+\rho_{f}(1-\phi) \boldsymbol{g} .}
\end{gathered}
$$

Here, $\rho_{s}$ and $\rho_{f}$ denote the densities of the particle and fluid phases, respectively; $\boldsymbol{v}$ and $\boldsymbol{u}$ are the local-average particle and fluid phase velocities; $\phi$ is the local particle volume fraction; $\boldsymbol{g}$ is specific gravitational force; $\boldsymbol{F}$ is the total fluid-particle interaction force (exerted by the fluid on the particles) per unit volume of the mixture; $\sigma_{s}$ and $\sigma_{f}$ are the effective particle and fluid phase stress tensors. The total fluidparticle interaction force is usually partitioned into two (e.g. see Lovalenti \& Brady 1993; Jackson 2000):

$$
\boldsymbol{F}=\phi \nabla \cdot \boldsymbol{\sigma}_{f}+\boldsymbol{f} .
$$

The first term on the right-hand side captures the effect of the slowly varying $\sigma_{f}$ field, while the second term accounts for the effects of the rapidly varying velocity and pressure fields around the individual particles. In what follows, $f$ will be referred to as the local fluid-particle interaction force per unit volume.

It is now well established that these equations coupled with simple phenomenological closures for $\boldsymbol{\sigma}_{s}, \boldsymbol{\sigma}_{f}$ and $\boldsymbol{f}$ can capture the experimentally observed structures in a qualitatively correct manner; however, quantitative predictions remain elusive (e.g. see Jackson 2000; Sundaresan 2003).

The interaction force $f$ is usually expressed as the sum of several components: the drag force $\left(f_{d}\right)$, added mass force $\left(f_{a}\right)$, history force $\left(f_{h}\right)$, etc. The drag force is written as:

$$
\boldsymbol{f}_{d}=\beta(\boldsymbol{u}-\boldsymbol{v}),
$$

where the drag coefficient $\beta$ depends on $\phi$, the local slip velocity, $|\boldsymbol{u}-\boldsymbol{v}|$, the particle size, and the physical properties of the fluid. For example, a constitutive relation of the form (Batchelor 1988; Jackson 2000),

$$
\beta=\frac{g}{v_{\infty}}\left(\rho_{s}-\rho_{f}\right) \phi(1-\phi)^{2-N},
$$

which can be deduced from the classic results of Richardson \& Zaki (1954), is widely used. Here, $v_{\infty}$ is the terminal settling velocity of a single particle in an unbounded fluid. The Richardson-Zaki exponent $N$ decreases monotonically with increasing particle Reynolds number based on $v_{\infty}, R e_{\infty}=v_{\infty} d_{p} / v$ (Richardson \& Zaki 1954; Batchelor 1988); here $d_{p}$ is the particle diameter and $v$ is the kinematic viscosity of the fluid. Much effort is in progress to develop more accurate closures through computational simulations (e.g. see Hill, Koch \& Ladd 2001; Li \& Kuipers 2003; Kandhai, Derksen \& Van den Akker 2003; Wylie, Koch \& Ladd 2003; van der Hoef, Beetstra \& Kuipers 2005). 
Developing closure relations for the effective stresses is considerably more difficult. The effective stress in the particle phase consists of several contributions: the streaming stress associated with the fluctuating motion of the individual particles; stress transmitted through particle-particle collisions; stress arising through other interactions between particles (e.g. electrostatic and van der Waals interactions); and a hydrodynamic component resulting from particle-particle interaction through the interstitial fluid. Closures for the effective stresses should also take into account the effects of the rates of compaction and dilation of the particle phase - which is typically captured through an isotropic contribution involving the particle phase bulk viscosity. The particle phase stress, $\boldsymbol{\sigma}_{s}$, is written as:

$$
\boldsymbol{\sigma}_{s}=-p_{s} \boldsymbol{I}+\kappa_{s}(\nabla \cdot \boldsymbol{v}) \boldsymbol{I}+\mu_{s}\left[(\nabla \boldsymbol{v})+(\nabla \boldsymbol{v})^{T}-\frac{2}{3}(\nabla \cdot \boldsymbol{v}) \boldsymbol{I}\right],
$$

where $p_{s}, \kappa_{s}$ and $\mu_{s}$ are the pressure, bulk viscosity and shear viscosity of the particle phase, respectively. The pressure and the viscosities are either closed through phenomenological models (for example, see Anderson et al. 1995; Duru et al. 2002) or are expressed through constitutive models based on the concepts of kinetic theory of dense gases. These kinetic theory of granular flow (KTGF) based models (for example, see Lun et al. 1984; Gidaspow 1994; Koch \& Sangani 1999; Agrawal et al. 2001) afford constitutive models for $p_{s}, \kappa_{s}$ and $\mu_{s}$ explicitly in terms of local particle volume fraction, $\phi, \rho_{s}, d_{p}$ and the fluctuating velocity of the particles. Although the actual expressions for the shear and bulk viscosities differ slightly from one derivation to another, all derivations yield comparable values for them, with the shear viscosity being larger in magnitude than the bulk viscosity (e.g. see Gidaspow 1994).

In the present study, we examine the adequacy of such constitutive models for $\sigma_{s}$ and $f$, using one-dimensional waves in fluidized beds as the model problem. Such travelling waves are made up of regions where the particle assemblies undergo dilation and regions where they compact. As compaction and dilation of particle assemblies are ubiquitous in granular and fluid-particle flows, it is important to test and validate constitutive models through clean model problems where both compaction and dilation occur.

Duru et al. (2002) have reported the particle volume fraction profiles in fully developed one-dimensional travelling waves in liquid-fluidized beds. These authors have also probed the variation of particle phase pressure and viscosity with $\phi$ from the experimentally measured particle volume fraction profile, the two-fluid model equations (1)-(6) and (8), and an assumed constitutive model for the drag coefficient. They found that

$$
\mu_{t}(\phi)=\mu_{s}(\phi)+\kappa_{s}(\phi) \approx 0.18 \frac{\rho_{s} d_{p} v_{\infty}}{\phi_{r l p}-\phi}, \quad \frac{\mathrm{d} p_{s}}{\mathrm{~d} \phi} \approx 0.2 \rho_{s} v_{\infty}^{2} \quad \text { or } \quad \frac{\mathrm{d} p_{s}}{\mathrm{~d} \phi} \approx 0.7 \rho_{f} v_{\infty}^{2} .
$$

Here, $\phi_{r l p}$ is the particle volume fraction at random loose packing. More critical assessment of the closure relations requires detailed data on the spatial variation of particle and fluid velocity fields, collision statistics, etc. in these travelling waves, in addition to the particle volume fraction profiles. These are not easily measured in dense suspensions; to date, such measurements have not been made. However, computer simulations can be used to obtain the missing data. In the present study, we have performed detailed simulation of the flow of the fluid and the particles in these travelling waves and extracted all the detailed data required for critical evaluation of the constitutive models. We have used the experimental data of Duru et al. (2002) to validate the computer simulations, so that some degree of confidence can be associated with the detailed data extracted from them. 
In our simulations, we explicitly resolve the motion of a set of spherical uniformly sized solid particles and the interstitial fluid. The fluid flow is simulated by means of the lattice-Boltzmann method (LBM), see, for example, Chen \& Doolen (1998). Other methods that have been reported in the literature for directly simulating the dynamics of dense suspensions with moving particles and resolution of the solid-liquid interface are distributed Lagrange multipliers (Pan et al. 2002; Singh, Hesla \& Joseph 2003), and smoothed particle hydrodynamics combined with the discrete element method (Potapov, Hunt \& Campbell 2001).

In this article, we first demonstrate that by numerical simulation we can qualitatively capture the wave phenomena observed by Duru et al. (2002). The body force due to gravity, hydrodynamic forces (stemming from the LBM, and subgrid-scale lubrication), and hard-sphere collisions govern the dynamics of the particles. From our simulations, we determine how the particle volume fraction, fluid and particle velocities, fluid-particle interaction force, and the various contributions to the stresses vary with position along the travelling wave. Such detailed information allows us to examine the importance of various terms in continuum models and various microphysics such as lubrication forces. Equally importantly, it allows us to assess whether closures developed from simulations of homogeneous suspensions should be supplemented for effects of compaction or dilation occurring in inhomogeneous systems.

In the subsequent sections, we will demonstrate that the inhomogeneous travelling structures seen in the experiments of Duru et al. (2002) and Duru \& Guazzelli (2002) readily evolve in the simulations, and that the propagation velocities obtained in the simulations are comparable to the experimental values. The simulations reveal that the rates of compaction and dilation of the particle phase in the void region of the travelling waves are comparable to the rate at which the microstructure relaxes, and that the rate of compaction of the particle assembly has a pronounced effect on the collisional stress in the particle phase.

The rest of the paper is organized as follows. In $\S 2$, we describe the specific cases in the experiments by Duru et al. (2002) which are simulated in our study. The numerical procedure is outlined in $\S 3$. The results are presented in $\S 4$ and the main findings of this study are summarized in $\S 5$. More detailed accounts have been placed in the Appendices.

\section{Flow system}

Duru et al. (2002) carried out an extensive study of the onset and characteristics of planar waves in relatively narrow liquid fluidized beds. Their experimental variables were the ratio of particle and fluid densities, the particle size, the fluid viscosity, the size ratio (particle diameter divided by tube diameter) and the average particle volume fraction (which was controlled by the superficial velocity of the fluidizing liquid). Even though planar waves formed spontaneously, they excited specific wave frequencies by subjecting the distributor plate to an oscillatory motion so that clean high-quality data could be obtained; therefore, the excitation frequency and amplitude were also inputs in the experiments. Figure 1 shows a typical experimental result obtained by them: a space-time plot of the solids volume fraction. Clearly visible are regions of low particle volume fraction ('voids') that travel with a well-defined speed in the vertical $(z)$ direction. Figure 2 shows two of the many waveforms measured in their experiments. Travelling waves are shown with a single hump (figure $2 a$ ) and two humps (figure $2 b$ ). These data were obtained by averaging over a hundred waveforms. 


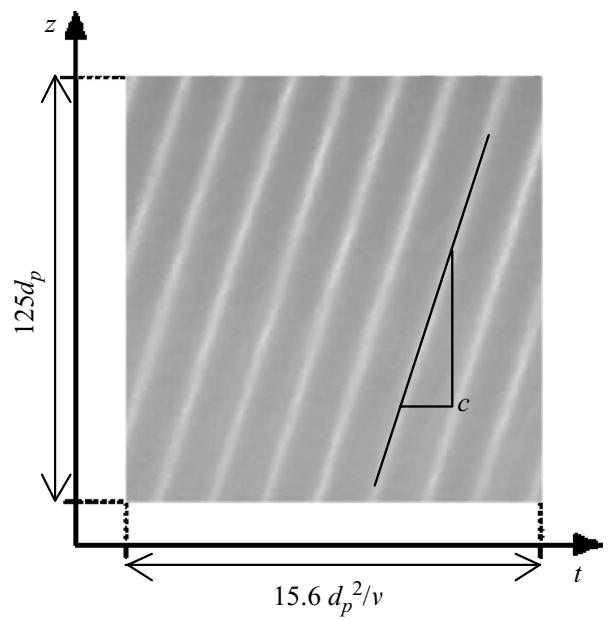

FiguRE 1. Experimental space-time plot of the solids volume fraction at $\bar{\phi}=0.540$. The wave speed $(c)$ can be derived from the slope of the light lines representing the void regions. [Reprinted from Duru et al. 2002.]

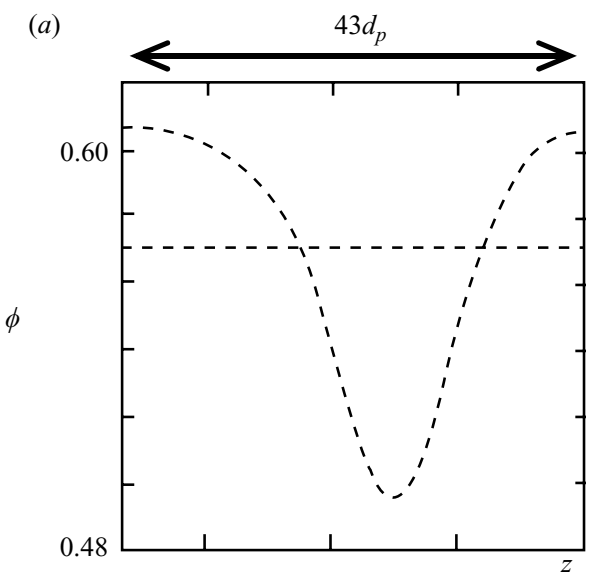

(b)

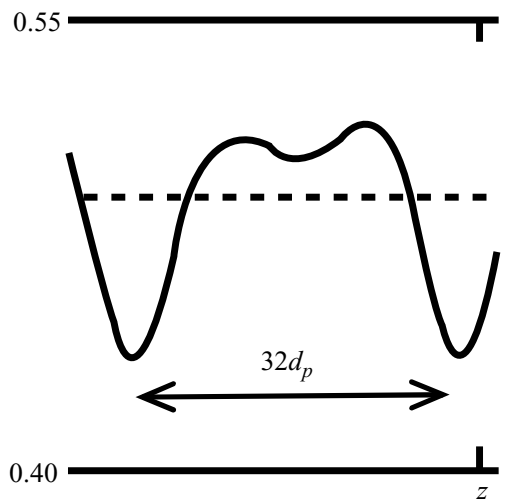

FiguRE 2. Experimental solids volume fraction wave profiles for $(a) \bar{\phi}=0.57$ and (b) $\bar{\phi}=0.49$. [Reprinted from Duru et al. 2002.]

In this paper, we first simulate one-dimensional travelling waves such as those shown in figures 1 and 2. The specific experiments that we selected from Duru et al. (2002) had particles with $d_{p}=685 \pm 30 \mu \mathrm{m}$, a density ratio $\rho_{s} / \rho_{f}=4.1$, and a fluid kinematic viscosity of $v=0.90 \times 10^{-6} \mathrm{~m}^{2} \mathrm{~s}^{-1}$ (these settings being denoted 'Combination 7 ' by Duru et al.).

In order to keep the computations affordable, we restrict our simulations to a fully-periodic three-dimensional domain. Since we wish to simulate one-dimensional travelling waves, we suppress the onset of secondary instabilities by choosing the lateral dimensions to be small (typically $6 d_{p}$ ). By repeating some of the simulated cases for smaller lateral domains $\left(5 d_{p}\right.$ and $\left.4 d_{p}\right)$, we evaluate the impact the domain size has on the results. It will become apparent that the fluid-particle drag is sensitive to the size of the domain. Most of the analysis in this paper will be devoted to a study of one-dimensional travelling waves. Towards the end of this paper, we present the 
results of a few simulations with wider periodic domains, illustrating the evolution of two-dimensional structures, as a further validation of the numerical approach employed in our studies.

In our simulations, we represent the experimental system by a set of uniformly sized spherical particles immersed in a fluid. The flow is induced by a force in the negative $z$-direction acting on the particles (gravity), and an effective body force acting on the fluid that balances the gravity force on the particles. This body force on the fluid is equivalent to the average pressure gradient (in laboratory experiments) that drives the flow of the fluidizing liquid up through the particle assembly. The total force acting on the fluid-particle mixture in the periodic domain is zero and so the total momentum of the mixture as a whole does not change with time in our simulations.

Simulations of one-dimensional travelling waves were performed in threedimensional periodic domains $\left(6 d_{p} \times 6 d_{p} \times 20 d_{p}\right)$, for several different average particle volume fractions $(\bar{\phi})$, but much of the discussion will be on results obtained for three specific $\bar{\phi}$ values $(0.580,0.505$ and 0.488$)$. The case with $\bar{\phi}=0.505$ will serve as our base case. Parametric sensitivity analyses - with respect to collision parameters, spatial resolution of the simulations and domain size - were performed around this base case.

The simulations differed from experiments in one important way: the frequency of the wave was controlled in the experiments by externally forcing the distributor plate, and the wavelength of the travelling structure emerged as an output. In contrast, the wavelength is fixed in the simulations and the resulting waveforms evolved spontaneously without any external forcing. This difference, however, does not limit the value of the simulations; waves did evolve in the experiments spontaneously, but one does not have the luxury of forcing a specified wavelength in the experiments although this is straightforward in the simulations.

\section{Numerical set-up}

In our simulations, we consider a three-dimensional (Cartesian) domain which is discretized into a number of lattice nodes residing on a uniform cubic grid. In the LBM, fluid parcels move from each node to its neighbours according to prescribed rules. It can be proved by means of a Chapman-Enskog expansion that, with the proper grid topology and collision rules, this system obeys, in the low-Mach-number limit, the incompressible Navier-Stokes equations (e.g. see Chen \& Doolen 1998). The specific implementation used in our simulations has been described by Somers (1993) and Eggels \& Somers (1995), which is a variant of the widely used lattice BGK scheme to handle the collision integral (e.g. see Qian, d'Humieres \& Lallemand 1992). We use in the results presented below the scheme due to Eggels \& Somers (1995), as it manifests a more stable behaviour at low viscosities when compared to LBGK. This scheme is second-order accurate in space and time.

Ladd $(1994 a, b)$ pioneered the application of lattice-Boltzmann methods for suspension simulations; since then, lattice-Boltzmann methods have been applied extensively for suspension simulations (e.g. Aidun, Lu \& Ding 1998; Ladd 1997; Hill, Koch \& Ladd 2001; Wylie et al. 2003; Ten Cate et al. 2004; Ten Cate \& Sundaresan $2006 a, b$ ). In our code, the no-slip condition at the solid-fluid boundaries is introduced through a forcing scheme (Goldstein, Handler \& Sirovich 1993; Derksen \& Van den Akker 1999; Ten Cate et al. 2002). The forcing scheme is akin to immersed boundary methods that have been developed by, among others, Peskin and co-workers (Griffith \& Peskin 2005). In this scheme, fluid is present everywhere in the periodic 
domain - both at the lattice nodes outside the solid particles ('true or external fluid') and at the nodes inside the solid particles ('fictitious or internal fluid'). Both external and internal fluid experience and respond to the effective fluid phase body force mentioned earlier. The fluid flow and the particle motion are coupled by demanding that at the surface of the sphere the fluid velocity matches the local velocity of the solid surface (that is the sum of the linear velocity $\boldsymbol{v}_{p}$ and $\boldsymbol{\Omega}_{p} \times\left(\boldsymbol{r}-\boldsymbol{r}_{p}\right)$ with $\boldsymbol{\Omega}_{p}$ being the angular velocity of the particle); in the forcing scheme this is accomplished by imposing additional forces on the fluid at the surface of the solid sphere (which is then distributed to the lattice nodes in the vicinity of the particle surface). The details of the implementation can be found elsewhere (Goldstein 1993; Derksen \& Van den Akker 1999; Ten Cate et al. 2002). The collection of forces acting on the fluid at the sphere's surface and its interior is subsequently used to determine the hydrodynamic force and torque acting on the sphere (action $=-$ reaction); see Appendix $\mathrm{A}$ for additional details.

In our simulations, the diameter of each spherical particle is specified and input diameter refers to this diameter scaled by the lattice spacing. In the LB simulations, as the spherical particle is represented by forces that are confined to a cubic grid, the input diameter does not reflect the actual diameter of the particle. A calibration procedure to estimate the effective diameter of this object (commonly referred to as the hydrodynamic diameter) was introduced by Ladd (1994a). We apply this scheme to estimate the hydrodynamic diameter of the particles. The hydrodynamic diameter is recognized as the diameter $d_{p}$ mentioned earlier.

The procedure employed to translate the physical parameters of the experiments into LB-parameters is straightforward. In lattice units, the spacing $\Delta$ and time step $\Delta t$ are taken as unity. The hydrodynamic diameter in lattice units $a$ is then equal to $d_{p} / \Delta$. The spatial resolution of the simulations can be expressed in terms of the magnitude of $a$. The default value of $a$ in our simulations was 16; results of sensitivity tests with respect to the parameter $a$ are discussed below. The fluid density (in lattice units) was nominally equal to 8 and the solid density was then chosen to obtain the desired density ratio. The average particle volume fraction in the periodic domain, $\bar{\phi}$, determined the number of particles in the domain. The only other dimensionless group to be matched between the experiments and simulations is $g d_{p}^{3} / v^{2}$. We demanded that $\left(g d_{p}^{3} / v^{2}\right)_{\exp }=g_{L B} a^{3} / \nu_{L B}^{2}$ and also insisted that the terminal settling velocity of a single particle in unbounded fluid be of the order of 0.02 in LB units. The latter condition assures that fluid velocities stay well below the speed of sound of the numerical scheme so that incompressible flow is being simulated.

In order to demonstrate that the above procedure represents the dynamics of spheres immersed in liquid properly, we considered the transient motion of a single sphere that is accelerated from rest under the influence of gravity. The equations describing the motion for an isolated sphere in an unbounded fluid at low Reynolds numbers are presented by Maxey \& Riley (1983). Results obtained by integrating the Maxey \& Riley equation are compared to our numerical results in figure 3 in terms of the time series of the velocity of the sphere. The simulation conditions were such that the Reynolds number based on the steady state settling velocity was 0.1 . Note that good agreement of the two solutions is found even at density ratios close to one. Ten Cate et al. (2002) compared the results on sedimentation of a single sphere in a finite-size container at higher Reynolds numbers (up to $R e=30$ ) obtained through lattice-Boltzmann simulations and forcing boundary conditions with particle image velocimetry (PIV) data, showing good agreement in terms of the sphere's trajectory and the fluid flow field around the moving sphere. 

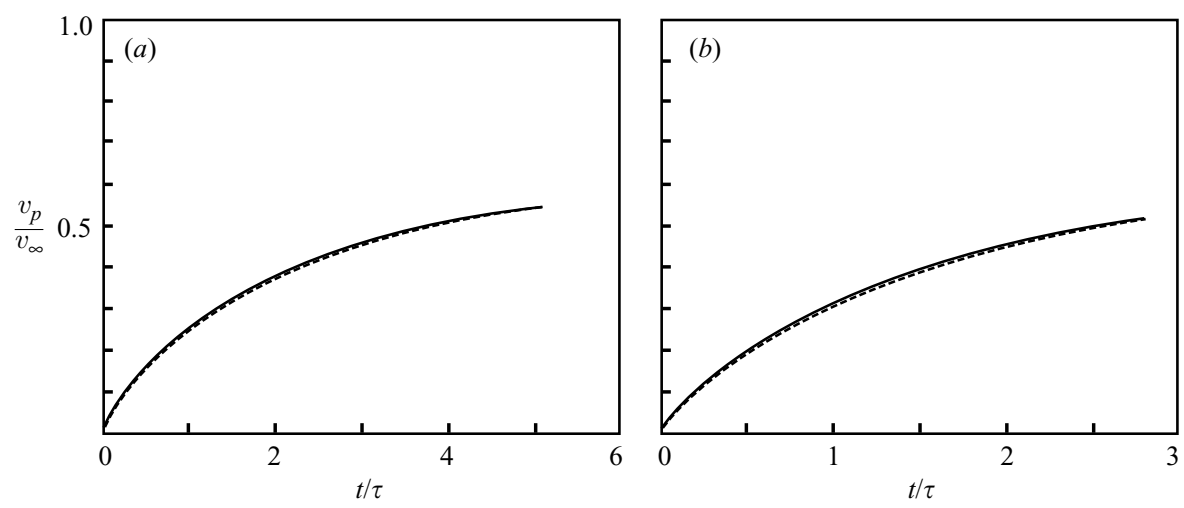

FIGURE 3. Time series of the velocity of a sphere falling under gravity at low Reynolds number. Solid curve: solution of the Maxey and Riley (1983) equation of motion; dashed curve: LB solution with a sphere with a hydrodynamic diameter of 16 lattice units. $(a) \rho_{s} / \rho_{f}=1.1$, (b) 2.0. The dashed and solid curves can hardly be distinguished. $v_{\infty}$ is the steady-state velocity; $\tau=\rho_{s} d_{p}^{2} / \rho_{f} 18 v$.

In our suspension simulations, we also take into consideration the interaction between particles through binary hard-sphere collisions and lubrication forces. For the former, we apply an event-driven collision algorithm: we move the collection of particles until two particles are in contact. At that moment, we carry out the collision (i.e. update the velocities of the two particles taking part in the collision). Subsequently, the movements of all particles are continued until the next collision or until the end of an LB time step. The collision model that we apply (Yamamoto et al. 2001) has two parameters: a restitution coefficient $e$ and a friction coefficient $\mu$. As the default situation, we consider fully elastic frictionless collisions $(e=1, \mu=0)$. We will also present results with different settings for $(e, \mu)$ to check their influence on the wave speed and the waveforms.

When two particles are in close proximity, with a separation of the order of or less than the lattice spacing, the hydrodynamic interaction between them will not be properly resolved in the LB simulations. Therefore, we explicitly impose lubrication forces on the particles, in addition to the hydrodynamic forces stemming from the LBM; see Appendix B for details of the implementation and validation.

The time-step-driven (LBM) and the event-driven (collisional) parts of the simulation have been combined by first performing the LBM time step from $t$ to $t+\Delta t$ and subsequently moving the particles until the particle system has advanced $\Delta t$ in time. Since in dense systems usually more than one collision occurs during $\Delta t$, the particle motion algorithm sets a number of sub-time-steps, the number being equal to one plus the number of (potential, see below) collisions.

At the start of every particle motion sub-time-step, we update the lubrication forces and torques. Then we move the particles over the sub-time-step, i.e. until the next potential collision (or until $t+\Delta t$ is reached). At the new positions of the particles, we again determine the lubrication force and torque. The linear and angular velocities of the particles are now updated according to the average of the lubrication forces and torques at the beginning and at the end of the sub-time-step. The velocity update may result in the collision not occuring: in that situation, the lubrication forces were strong enough to change the sign of the relative particle velocity so that a hard-sphere collision was prevented. 


\section{Results}

\subsection{Waves and wave speeds}

A homogeneous bed was first created by placing a set of non-overlapping spheres randomly in space in the periodic domain. Random packings up to $\bar{\phi} \approx 0.3$ can be prepared in this manner (Torquato, Truskett \& Debenedetti 2000). Higher levels of $\bar{\phi}$ were reached by letting the system collapse under the influence of a body force. After the desired solids volume fraction was obtained, the system was equilibrated by giving the spheres random velocities and moving them around in the periodic box as a granular gas for some time. (In such a granular gas simulation, the motion of the particles is not affected by the interstitial fluid, and they only respond to elastic collisions with other particles.) At the start of the two-phase-flow simulation, the velocities of the particles and the fluid were set to zero. At $t=0$, gravity and the body force on the fluid were turned on. As the lateral dimensions of the domain are small $\left(6 d_{p}\right)$, there is little opportunity for any persistent lateral structure to evolve, but one can readily see non-uniform structures that travel in the direction of the mean fluid flow. Figure 4, shows a series of snapshots taken at an arbitrary vertical cross-section of the periodic domain. The system develops a wave structure in a time span of the order of $d_{p}^{2} / \nu$. A region of lower particle volume fraction, hereinafter referred to as 'void', travels in the opposite direction to gravity (i.e. the positive $z$-direction). Outside the void, the particle volume fraction is significantly higher than the average value, $\bar{\phi}$. Above the void, particles detach from the dense region, then 'rain' through the void, and subsequently collect on the dense region below the void.

The simulated wave was averaged over the horizontal directions ( $x$ and $y$ ), and represented in a space-time plot similar to the experimental one (see figure 1). Examples of such plots are given in figure 5. The wave amplitude and structure depend on the average particle volume fraction, $\bar{\phi}$ : shallow waves form at high $\bar{\phi}$, whereas more complicated waves form for lower $\bar{\phi}$ values. The dimensionless wave speeds $\tilde{c}=c d_{p} / v$ (with $c$ being the dimensional wave speed) extracted from figure 5 are $30.9,33.7$ and 33.2 for $\bar{\phi}=0.580,0.505$ and 0.488 , respectively. The error margin in determining $\tilde{c}$ from the simulation results was estimated as \pm 1 .

The wave speed decreased slowly, but monotonically, as the wavelength of the domain was increased. For $\bar{\phi}=0.505$ and $a=16$, the dimensionless wave speeds $\tilde{c}$ obtained in simulations with wavelengths of $10 d_{p}, 20 d_{p}$ and $40 d_{p}$ were $36.0 \pm 1$, $33.7 \pm 1$ and $29.4 \pm 1$, respectively.

Duru et al. (2002) measured dimensionless wave speeds of $\tilde{c}=28$ and $29( \pm 1.4)$ for their 'Combination 7' system at $\bar{\phi}=0.488$ and 0.496 , respectively; the corresponding wavelengths were $\sim 26 d_{p}$ and $\sim 36 d_{p}$. The wave velocities derived from our simulations deviate only slightly from the experimental data. This difference is perhaps related to the flow resistance offered by the tube wall in the experiments, which is missing in the simulations.

By varying numerical parameters, while keeping the dimensionless numbers that govern the flow system constant, we evaluated the consistency of our results for our base case : $\bar{\phi}=0.505$ and wavelength (i.e. height of our simulation domain) $=20 d_{p}$. An increase of the acceleration due to gravity by a factor of 4 , and of the viscosity by a factor of 2, while keeping the rest of the parameters constant, typically resulted in fluid and particle velocities that were twice as high. The wave that developed in this system had a speed that was twice as high. The dimensionless wave speed $\tilde{c}$, however, remained practically unchanged. Apart from consistency, this test indicates that our choice for the velocity scale in the base case (which was based on a single particle 
(a)

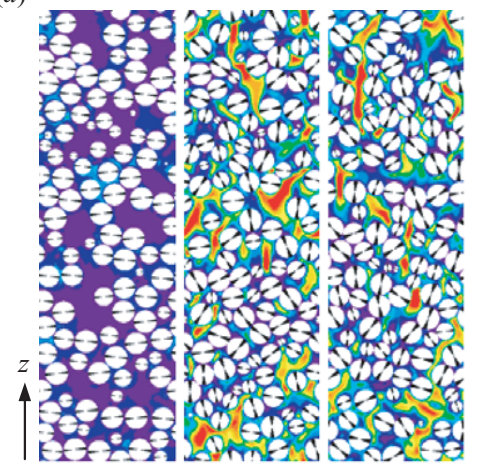

(b)

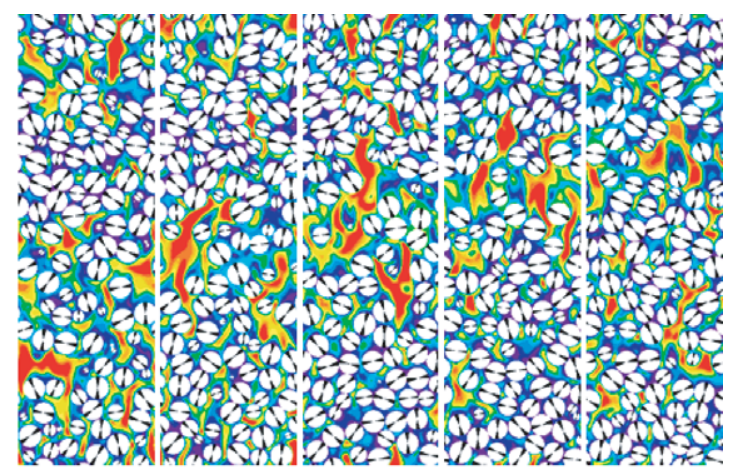

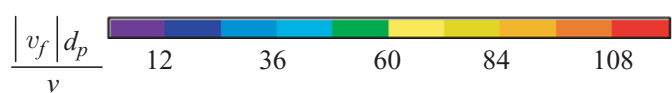

FIGURE 4. Cross-sections through the simulated solid-liquid field with $\bar{\phi}=0.505$ at various moments in time. $(a)$ The initial stages of the simulation. $(b)$ The wave travelling at constant speed. (From left to right $t v / d_{p}^{2}=0.026,0.55,1.095,1.225,1.277,1.329,1.381,1.434$.) The colours denote the absolute value of the fluid velocity. All the spherical particles have the same size.

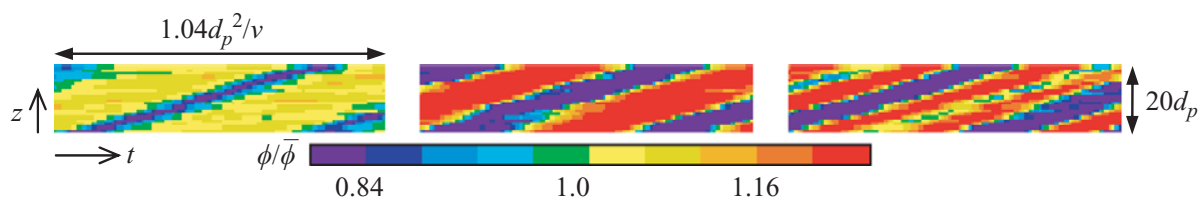

FigURE 5. Simulated space-time plots of the solids volume fraction. From left to right: $\bar{\phi}=0.580,0.505$ and 0.488 . Wavelength (= height of our simulation domain) $=20 d_{p} . a=16$.

falling with a terminal velocity of 0.02 in LB units) was appropriate with a view to compressibility effects in the LBM.

The dimensionless wave speed appears to be only weakly sensitive to the grid resolution. If we compare the wave speed of our base case (with resolution parameter $a=16)$ with simulations that had $a=12$ and $a=24$, the wave speeds we find are $\tilde{c}=34.4(a=12) ; \tilde{c}=33.7(a=16) ; \tilde{c}=32.5(a=24)$.

A fully developed wave, when viewed in a co-travelling frame, is clearly a steady solution. The data gathered in our simulations, when viewed in such a co-travelling frame, represent various realizations of the travelling-wave solution. By ensemble-averaging over all these realizations, we determined the axial profiles of various macroscopic quantities in the co-travelling frame. In every simulation, after establishing a fully developed wave, data sets containing short-time averages (averaging time $t_{a}=5.2 \times 10^{-4} d_{p}^{2} / v$ corresponding to $50 \mathrm{LB}$ time steps) of volume fraction, velocities and other relevant quantities, were computed as functions of the vertical location in the wave. We have summarized in Appendix $C$ the numerical procedure used to compute these quantities. A series of 2000 such data sets (spanning a time $2000 t_{a}$; in this time span the wave typically travelled $30 d_{p}$ ) were used to determine the ensemble-averaged profiles in the co-travelling frame. In what follows, all the quantities are presented and analysed in this co-travelling frame.

The particle volume fraction profiles shown in figure 6 are analogous to those reported by Duru et al. (2002). The simulated profiles certainly resemble the measured 

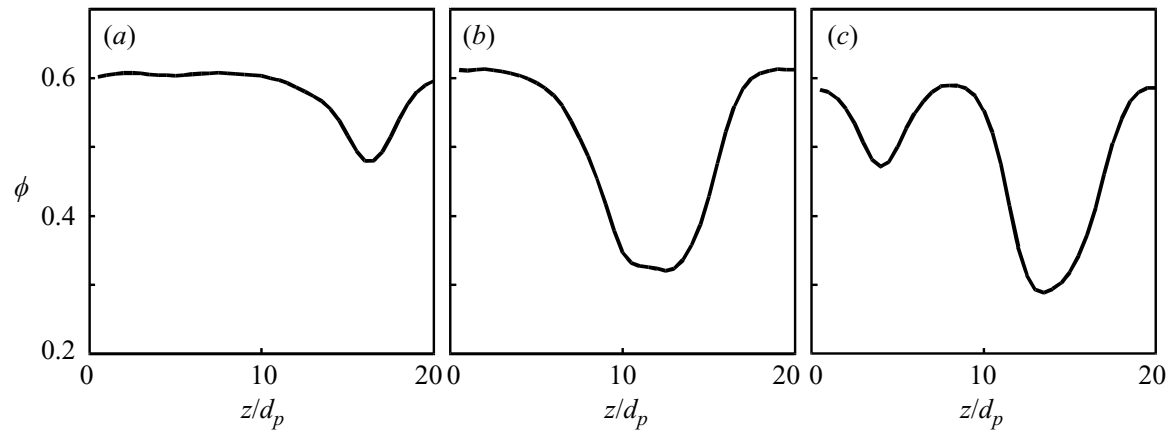

FIGURE 6. Solids volume fraction wave profiles for (from left to right) $\bar{\phi}=0.580,0.505$ and 0.488. $a=16$.
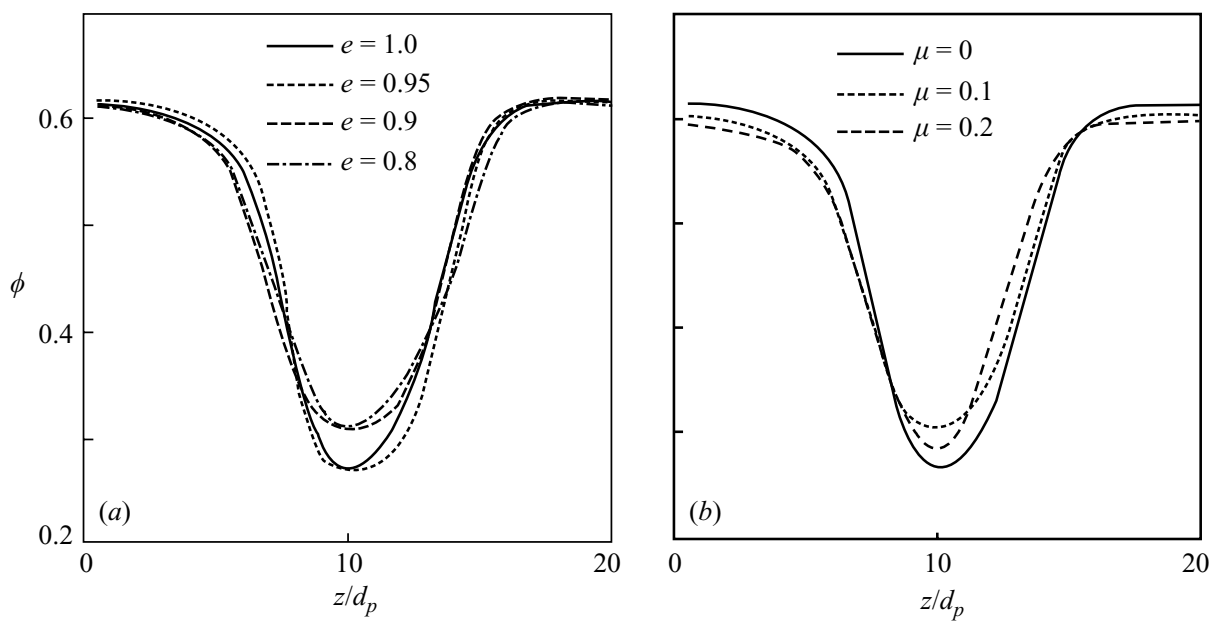

FiguRE 7. Wave shapes for various collision parameters at $\bar{\phi}=0.505$. (a) Profiles for different values of the restitution coefficient at $\mu=0$. $(b)$ Profiles for different values of the friction coefficient at $e=1.0$. The wave profiles were horizontally shifted such that the minimum values coincided. $a=16$.

ones; it is striking that even the two-humped wave seen in the experiments at lower $\bar{\phi}$ values are captured in the simulations. Thus, we believe that the experimental data of Duru et al. (2002) provide a qualitative validation of our simulations. We did not attempt detailed quantitative comparison as the waves obtained in our simulations were 'unforced', whereas those generated by Duru et al. (2002) were externally 'forced', as discussed earlier.

In the above simulations, the hard-sphere collisions were assumed to be fully elastic and frictionless $(e=1, \mu=0)$. We probed the effects of inelasticity and friction on the wave speed and the wave shape. The wave formed readily in every case. The wave speeds for $(e, \mu)=(1.0,0.0),(0.95,0.0),(0.8,0.0),(1.0,0.2)$ were essentially indistinguishable. The wave shape depended only weakly on the collision parameters (figure 7). Thus, the deviations between measured and simulated wave speeds are not due to uncertainties in the collision parameters. The energy dissipated in the inelastic collisions is very small. In the $(e, \mu)=(0.95,0.0)$ case, only $0.8 \%$ of the power inserted in the system is dissipated in the collisions, while lubrication dissipates $\sim 3 \%$. The rest is dissipated in the resolved fluid motion. 


\subsection{Quantitative analysis: fluid-particle interaction force}

Hill et al. (2001), Kandhai et al. (2003), van der Hoef et al. (2005) and Ten Cate \& Sundaresan $(2006 a, b)$ used static arrays of spheres and measured in series of simulations the effects of the solids volume fraction, Reynolds number and sphere configuration on the drag coefficient. Wylie et al. (2003) considered homogeneous assemblies of massive particles executing random motion in a periodic box and exposed the effect of the mean-squared fluctuating velocity of the assembly of particles on the drag coefficient. In our simulations, the inhomogeneity associated with the wave provides us with data on fluid-particle interaction force over a range of solids volume fractions in a single simulation.

The total fluid-to-particle force per unit volume of the suspension, $\boldsymbol{F}=\boldsymbol{F} \boldsymbol{e}_{z}$, along the wave is readily found from the simulation results. The local fluid-particle interaction force, $\boldsymbol{f}=f \boldsymbol{e}_{z}$, is then found (see Appendix D):

$$
f=F+\phi \frac{\mathrm{d}}{\mathrm{d} z}\left(p+\sigma_{f s, z z}\right),
$$

where $\sigma_{f s, z z}$ is the $z z$-component of the fluid phase streaming stress. To assess the role of the fluid phase streaming stress, we will consider an additional quantity

$$
f_{a}=F+\phi \frac{\mathrm{d} p}{\mathrm{~d} z} .
$$

We present below the variation of $F, f$ and $f_{a}$ along the wave.

The local hydrodynamic force component, $f$, is usually partitioned into several components: the drag force, added mass force, history force, etc. For the fully developed one-dimensional flow considered here, the added mass force can be written as

$$
C_{a} \rho_{f} \phi(1-\phi)\left[v_{f} \frac{\mathrm{d} v_{f}}{\mathrm{~d} z}-v_{p} \frac{\mathrm{d} v_{p}}{\mathrm{~d} z}\right]
$$

where $C_{a}$ is the added mass coefficient; $v_{p}$ and $v_{f}$ are the $z$-velocities of the two phases in this co-travelling frame. For isolated particles $C_{a}=0.5$ (Auton, Hunt \& Prud'homme 1988) and it differs only marginally for suspensions (Ten Cate \& Sundaresan 2006b). We can therefore use the above expression to estimate the potential contribution of the added mass force term to $f$ (and to $f_{a}$ ). We found that the added mass force estimated in this manner is no more than $0.4 \%$ of the force $f$. Ten Cate \& Sundaresan (2006b) found that the history-force and the added mass force contributions were comparable, and so we simply attribute the entire local hydrodynamic force to fluid-particle drag and write:

$$
f=\beta\left(v_{f}-v_{p}\right), \quad f_{a}=\beta_{a}\left(v_{f}-v_{p}\right),
$$

with $\beta\left(\beta_{a}\right)$ depending on the particle Reynolds number (based on the local slip velocity $\left.v_{f}-v_{p}\right)$, and the particle volume fraction. The wave profiles of the variables involved in (10)-(12) are presented in figure $8(a-c)$ for the base case. The dimensionless drag coefficient $\tilde{\beta}=\beta v / g\left(\rho_{s}-\rho_{f}\right) d_{p}$ is plotted against $z$ in figure $8 d$ and against the local $\phi$ in figure 8(e). Figure $8(b)$ shows that the fluid streaming stress term is small, however significant. Its most striking effect is a reduction (almost removal) of the path dependence of $\beta$ as a function of $\phi$ (compare figures $8 e$ and $8 f$ ): $\beta_{a}$ depends on the branch of the wave it is determined in, it has slightly higher values for the dilation branch as compared to the compaction branch. The coefficient $\beta$ as a function of $\phi$ does not show this ambiguity anymore: to a very good approximation, $\tilde{\beta}$ can be expressed as a function of $\phi$ alone. This is reminiscent of a widely used constitutive 

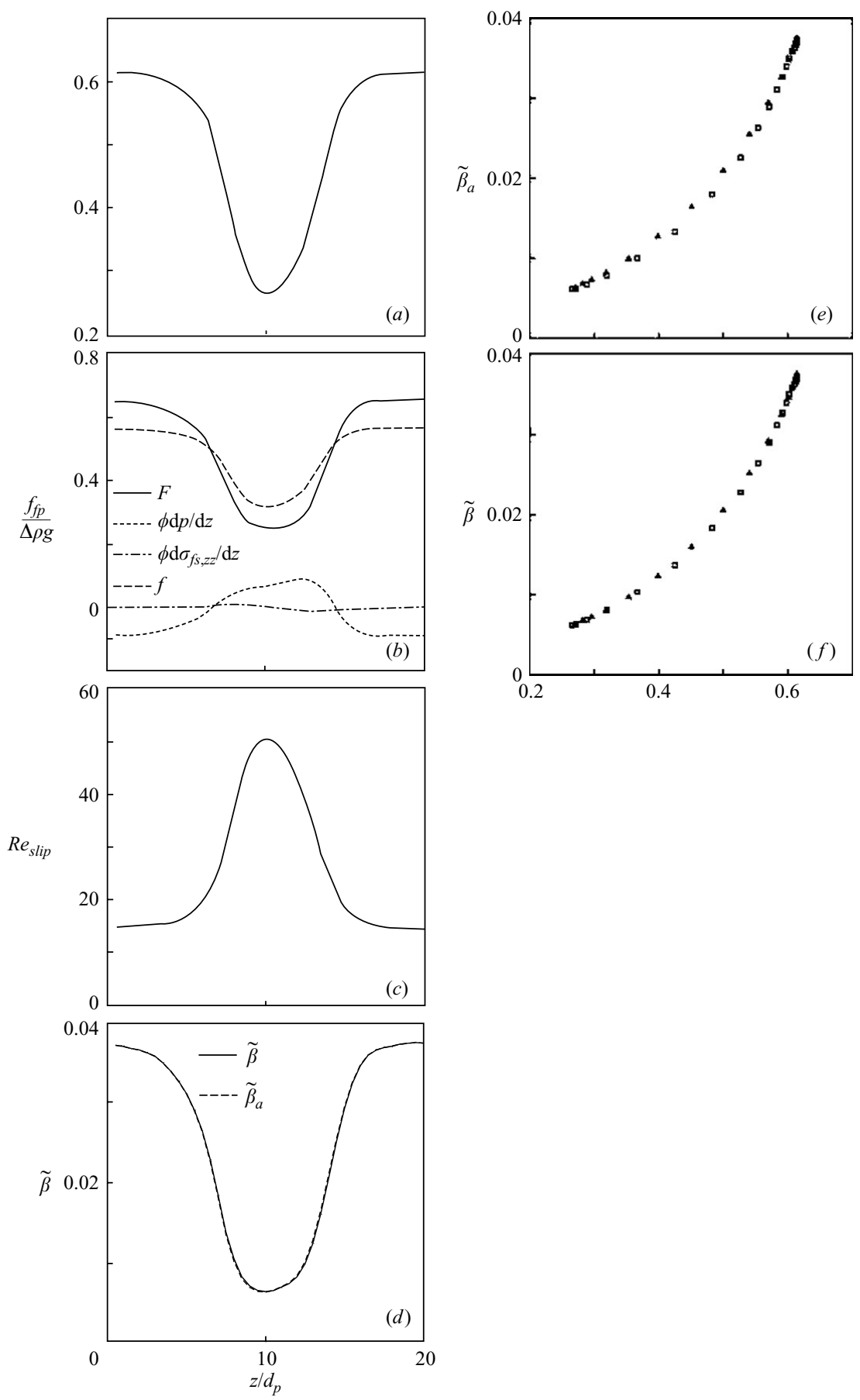

FiguRE 8. Determination of $\beta$ (equation (12)) along the wave for the base case. $(a) \phi$-profile, (b) fluid-to-particle forces (i.e. the various terms in equations (10) and (11)), (c) Reynolds number based on the slip velocity $R e_{\text {slip }}=\left|\boldsymbol{v}_{f}-\boldsymbol{v}_{p}\right| d_{p} / v$, (d) resulting $\beta$-profile where $\beta$ has been non-dimensionalized according to $\tilde{\beta}=\beta v / g\left(\rho_{s}-\rho_{f}\right) d_{p},(e, f) \tilde{\beta}$ versus $\phi$. We distinguish between $\tilde{\beta}_{a}$ and $\tilde{\beta}$ (equation (12)). The triangles denote that $\tilde{\beta}\left(\tilde{\beta}_{a}\right)$ was determined in the dilation branch of the wave, the squares that $\tilde{\beta}\left(\tilde{\beta}_{a}\right)$ was determined in the compaction branch. 


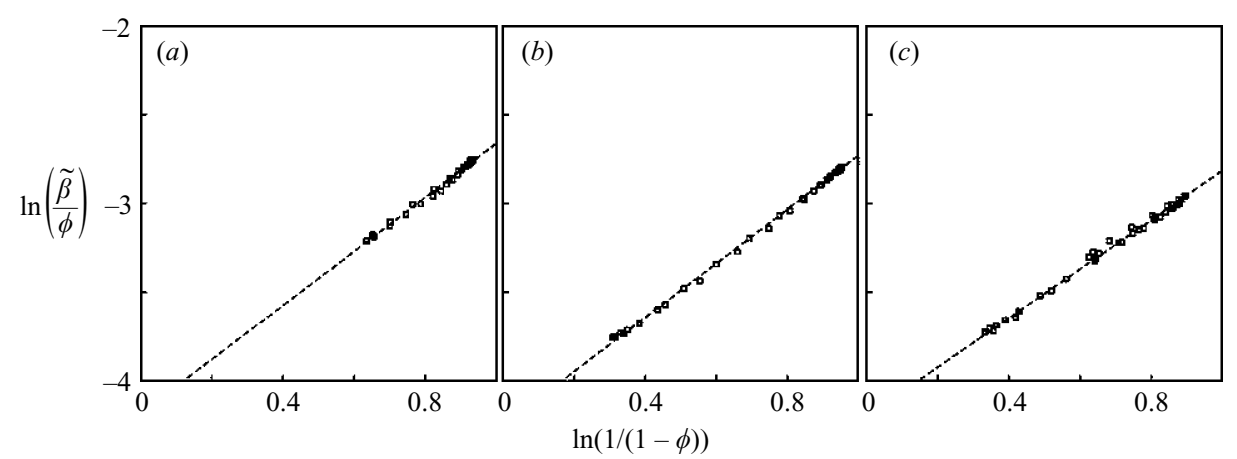

FIGURE $9 . \ln (1 /(1-\phi))$ versus $\ln (\tilde{\beta} / \phi)$ along with a linear fit, in the spirit of equation (13). (a) $\bar{\phi}=0.580,(b) 0.505$ and $(c) 0.488$. The slopes of the lines correspond to $N=3.49,3.49$ and 3.36 , respectively. The intercepts with the vertical axes are $-4.17,-4.23$ and -4.19 , respectively; these correspond to Reynolds numbers of 65, 68 and 66. $a=16$.

relation of the form presented earlier (see (7)). In terms of dimensionless variables, (7) takes the form:

$$
\tilde{\beta}=\frac{1}{R e_{\infty}} \phi(1-\phi)^{2-N} .
$$

The logarithmic plots in figure 9 lend some support to this type of closure model. The linear fits to the data points in figure 9 indicate $N \approx 3.5$. The most striking feature in this figure is in the intercept. According to (13), the intercept of the fitted lines with the vertical axes in figure 9 should be equal to $\ln \left(1 / R e_{\infty}\right)$. The values of $R e_{\infty, a p p}$ estimated from the intercepts in figure 9 are around 66, which is some $45 \%$ below $R e_{\infty}=120$. The latter value would follow from a force balance on a single particle and applying (for instance) the Schiller \& Naumann (1933) correlation for the drag coefficient $C_{D}=(24 / R e)\left(1+0.15 R e^{0.687}\right)$.

The origin of the difference is at least in part due to the limited domain size. Results of two additional simulations that differed from the base-case only in their lateral dimensions $\left(5 d_{p}\right.$ and $4 d_{p}$; whereas the base-case has $\left.6 d_{p}\right)$ showed that $R e_{\infty}-R e_{\infty, a p p}$ is roughly inversely proportional to the lateral domain size. Figure 10 shows the $\beta v s$. $\phi$ relation for the smaller domains. The dependence of $R e_{\infty, a p p}$ on the lateral domain size is presented in figure 11. An extrapolation to an infinitely wide domain would bring $R e_{\infty, a p p}$ close to $R e_{\infty}$.

\subsection{Momentum transfer}

The liquid-particle system transfers momentum through a variety of mechanisms: particle and fluid streaming motion (streaming stresses), particle-particle collisions (collisional stress), particle-particle interaction through lubrication forces, and fluidphase viscous stresses. The wave clearly induces anisotropy. As an example, we show in figure 12 the three components of the collisional normal stress. (In this figure, stresses have been non-dimensionalized according to $\tilde{\sigma}=\sigma d_{p}^{2} / \rho_{f} \nu^{2}$; this scaling of stresses (and pressure) has been applied throughout this paper, unless otherwise stated.) As expected, the two lateral components ( $x x$ and $y y$ ) are approximately equal to one another, and the axial component $(z z)$ differs appreciably from the other two. The effects of particle volume fraction are clearly visible in the stress profiles. The collisional stress is much lower in the void region than in the dense plug.

The most important $z z$-stresses are presented in figure 13. Collisions are largely responsible for the particle phase stress in the parts of the flow that have a high particle 


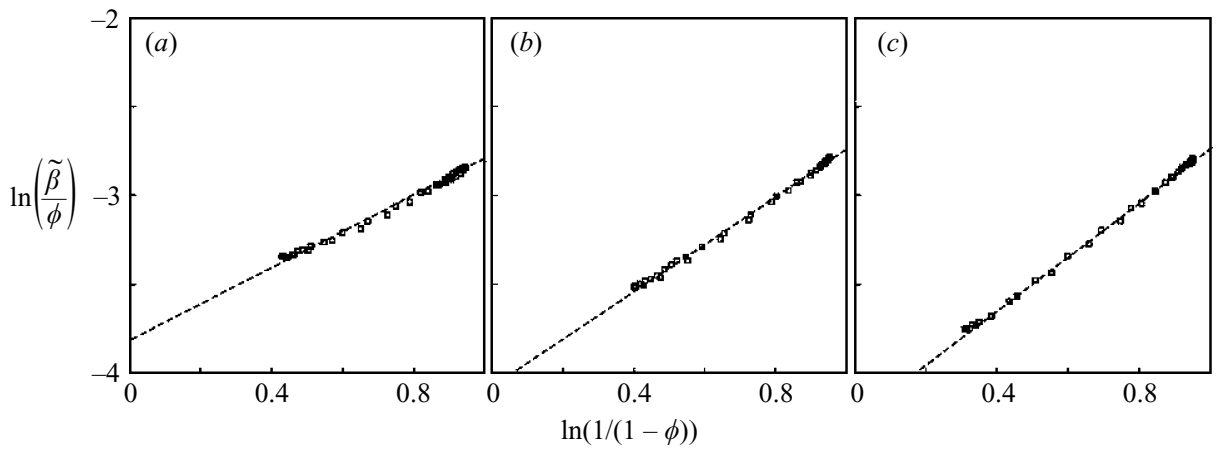

Figure $10 . \ln (1 /(1-\phi))$ versus $\ln (\tilde{\beta} / \phi)$ along with a linear fit for $\bar{\phi}=0.505$ with lateral domain size $(a) 4 d_{p},(b) 5 d_{p}$ and $(c) 6 d_{p}$ (same as figure $9 b$ ). Slopes of the fitted lines correspond to $N=3.06,3.36$ and 3.49 , respectively. The intercepts with the vertical axes are $-3.83,-4.09$ and -4.23 , respectively.

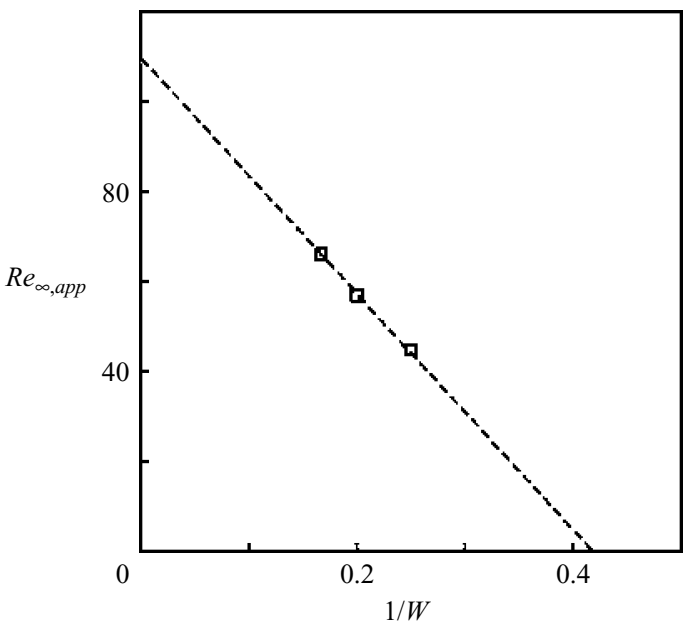

FIGURE 11. $R e_{\infty, a p p}$ as a function of the reciprocal lateral domain size $1 / W$ for the three cases given in figure 10, along with a linear fit line.
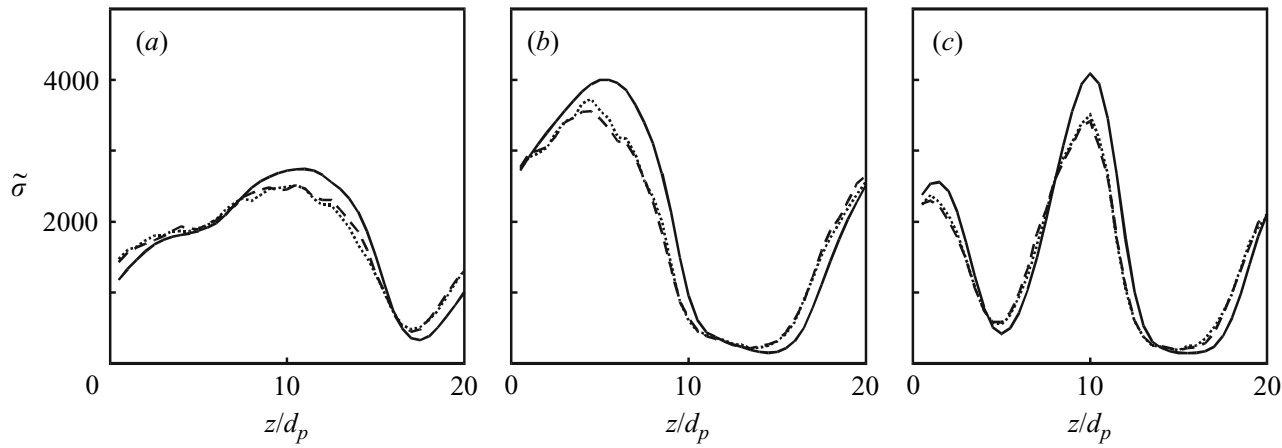

FIGURE 12. Variation of the three collisional normal stresses $(z z:$ drawn curve; $x x:$ dashed; $y y$ : dotted) along the wave. $\tilde{\sigma}$ is the dimensionless stress defined as $\tilde{\sigma}=\sigma d_{p}^{2} / \rho_{f} v^{2}$. (a) $\bar{\phi}=0.580$, (b) 0.505 and (c) 0.488. $a=16$. 

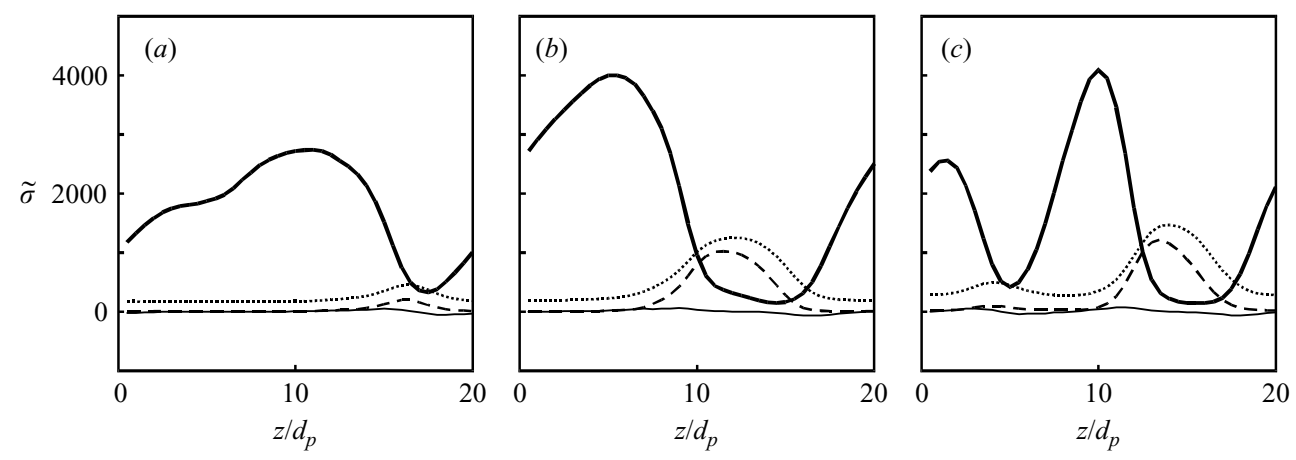

FIGURE 13. Variation of $z z$-stresses along the wave ( $\tilde{\sigma}$ has been defined in figure 12). (a) $\bar{\phi}=0.580,(b) 0.505$ and $(c) 0.488 . a=16$. Thick line: collisional stress; thin line: stress due to lubrication; dotted line: fluid streaming stress; dashed line: particle streaming stress.

volume fraction. In the void, fluid and particle streaming stresses are significant and of comparable magnitude. Lubrication plays only a modest role. The fluid-phase viscous normal stresses (not shown in figure 13) are negligible.

\subsection{Particle stresses in relation to Eulerian two-phase flow models}

The constitutive model for the particle phase stress, $\sigma_{s}$, commonly employed in two-fluid models was discussed earlier (see (8)). It is important to note that in all theories, the bulk and shear viscosities depend on local particle volume fraction and granular temperature (if included as a variable), but not explicitly on the local rate of deformation. At prescribed particle volume fraction and granular temperature, the particle phase stress depends linearly on the rate of compaction or dilation of the particle phase (which is captured through the $\nabla \cdot \boldsymbol{v}_{p}$ term); and the bulk and shear viscosities are independent of whether the assembly is undergoing compaction or dilation locally. Modifications of the closures to account for the presence of interstitial fluid have so far neither changed the relative magnitudes of the shear and bulk viscosities appreciably nor introduced a dependence of these quantities on the rate of compaction or dilation (Gidaspow 1994; Koch \& Sangani 1999; Brady, Khair \& Swaroop 2006).

In figure 14, we present the way the average collisional normal stress (defined as $\left.p_{c}=\left(\sigma_{c, x x}+\sigma_{c, y y}+\sigma_{c, z z}\right) / 3\right)$ varies with the local particle volume fraction. Note that $p_{c}$ denotes the sum of the contributions of collisions to the first two terms on the right-hand side of (8). Figure 14(a) and 14(b) take the form of a single lobe, as it corresponds to a single-hump wave, and it shows unequivocally that the average normal stress is not a unique function of particle volume fraction and that it is dramatically higher in the compaction branch than in the dilation branch. According to Wang \& Ge (2005), the kinetic theory of granular flows can yield good estimates, if it is supplemented with a good estimate of the velocity fluctuations. They proposed an energy budget analysis to estimate the particle velocity fluctuations and showed that the corresponding particle phase pressure is in good agreement with the data of Zenit et al. (1997). As our computations directly yield the granular temperature, we can evaluate the adequacy of the KTGF using the computed particle phase pressure and granular temperature. We show the ratio of collisional pressure over granular temperature in figure 15 which shows that the lobe structure is still retained illustrating that the average normal stress profile determined in our simulations cannot be simply identified with the collisional pressure of the KTGF. We have included in 

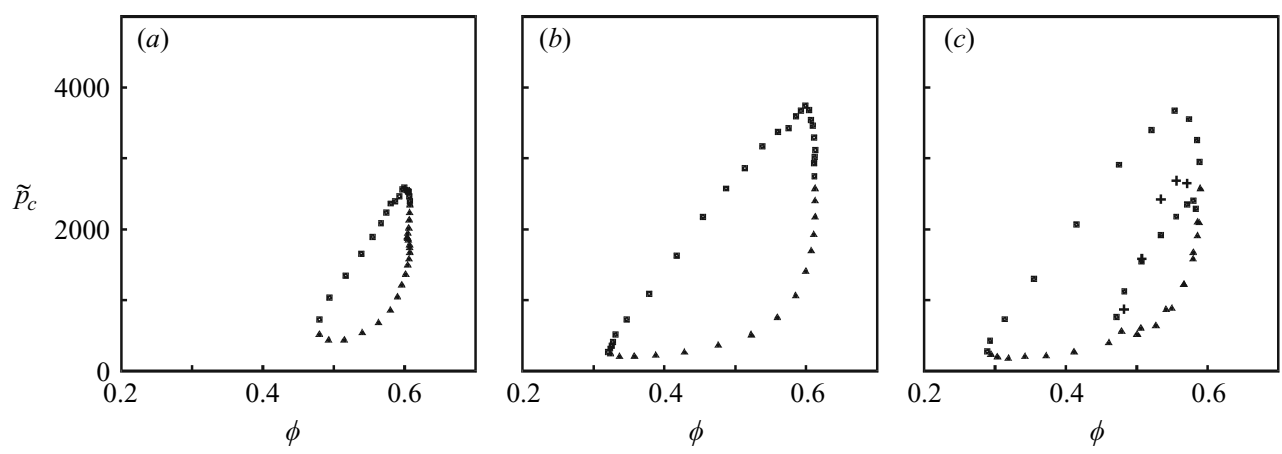

FiguRE 14. Dimensionless collisional pressure $\tilde{p}_{c}$ as a function of solids volume fraction. (a) $\bar{\phi}=0.580,(b) 0.505$ and (c) 0.488 . The squares (triangles) indicate data in the compaction (dilation) branch. The plus signs in $(c)$ are average collisional normal stresses in the shallow void based on bulk viscosity estimates from the deep void (equation (14)).

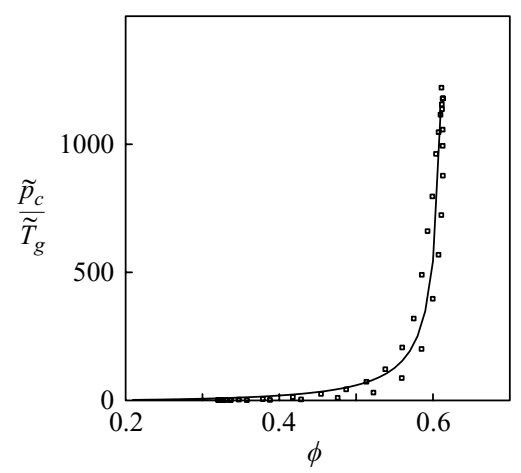

FiguRE 15. $\tilde{p}_{c} / \tilde{T}_{g} v s . \phi$ for the case with $\bar{\phi}=0.505$. Here, $\tilde{p}_{c}$ is the dimensionless collisional normal stress; $\tilde{T}_{g}=T_{g} d_{p}^{2} / v^{2}$ is the dimensionless granular temperature. Symbols: LB simulation; curve: KTGF estimate according to Wang \& Ge (2005).

figure 15 the magnitude of $p_{c} / T_{g}$ according to the KTGF (for fully elastic collisions):

$$
\frac{p_{c}}{T_{g}}=4 \rho_{s} \frac{\phi^{2}}{\left[1-\left(\phi / \phi_{r l p}\right)^{1 / 3}\right]}
$$

where we took $\phi_{r l p}=0.62$. It is clear that the computed results are not too far from the KTGF predictions, but for the lobe structure.

We determined the collisional pressure averaged over space and time for a range of average solid volume fractions with the default $20 \times 6 \times 6 d_{p}^{3}$ domain size in order to compare with the experimental results of Zenit et al. (1997), and with the model by Wang \& Ge (2005). The results are presented in table 1, where we have normalized the pressure with $\rho_{s} v_{\infty}^{2} / 2$, i.e. in the same way as Zenit et al. did. Note that for the LB simulations, we also could have normalized with $\rho_{s} v_{\infty, a p p}^{2} / 2$ which would have yielded values approximately three times higher; see the discussion around figures 9-11. The scatter in Zenit et al.'s experimental results is reflected in the ranges given in table 1 . In applying Wang $\&$ Ge's model we assumed $e=1$, and (as above) $\phi_{r l p}=0.62$.

The order of magnitude of the collisional pressure, and the trends as a function of the average solids volume fraction - slowly increasing (almost constant) pressure with increasing $\bar{\phi}$ up to $\bar{\phi}=0.5$ where the pressure quickly collapses - roughly agree with 


$\begin{array}{cccc}\bar{\phi} & \text { LB } & \text { Zenit et al. (1997) } & \text { Wang \& Ge (2005) } \\ 0.360 & 0.0186 & 0.03-0.1 & 0.08 \\ 0.420 & 0.0318 & 0.03-0.09 & 0.13 \\ 0.488 & 0.0501 & 0.02-0.08 & 0.16 \\ 0.505 & 0.0689 & 0.01-0.05 & 0.16 \\ 0.542 & 0.0612 & 0.003-0.02 & 0.16 \\ 0.580 & 0.0586 & - & 0.15 \\ 0.610 & 0.0212 & - & 0.063\end{array}$

TABLE 1. Average solids volume fraction $\bar{\phi}$ versus average dimensionless collisional pressure $\bar{p}_{c} / \frac{1}{2} \rho_{s} v_{\infty}^{2}$ for LB simulations (this work), according to experiments by Zenit et al. (1997), and according to the model by Wang \& Ge (2005).

Zenit et al.'s observations for $3 \mathrm{~mm}$ glass particles fluidized in water. The Reynolds number based on the terminal velocity in their experiment was $R e_{\infty}=954$, whereas in our simulations it was 120 . In the Wang \& Ge model, the collapse occurs at higher values of $\bar{\phi}$ and is less drastic as compared to the experiments. The LB simulations have a pressure that is a factor of 3 smaller than the pressure due to Wang $\& \mathrm{Ge}$ (2005); the uncertainty related to the normalization of the LB results is of the same order (see above).

Figure 14(c) shows two lobes as it corresponds to a double-hump wave. The rates of compaction and dilation in the shallower void (figure 12c) are much smaller than those in the deeper void; figure $14(c)$ suggests that the average collisional normal stress in the dilation branch is approximately independent of the rate of dilation. In contrast, the average collisional normal stress in the compaction branch of the shallower void is appreciably lower than that of the deeper void, indicating a pronounced dependence on the rate of compaction.

In the kinetic theory, particle phase pressure depends on both volume fraction and the granular temperature, and the granular temperature is indeed higher in the compaction branch than in the dilation branch, but this difference is no more than $30 \%$, and it cannot explain the factor of 4-6 difference seen in the average collisional normal stresses in the two branches at intermediate concentrations. It is natural to begin by suspecting that the path dependence seen in figure 14 is a consequence of the bulk viscosity effect, with the bulk viscosity being essentially zero under dilation and non-negligible upon compaction. In this line of thinking, the difference between the compaction and dilation branches in figure 14 is exclusively attributed to the bulk viscosity effect. To test this further, we used the data in the outer lobe of figure 14(c) and estimated the bulk viscosity at different particle volume fractions according to

$$
p_{c}=p_{s}-\kappa_{s} \frac{\partial v_{p, z}}{\partial z} .
$$

The reference pressure $p_{s}$ as a function of $\phi$ was estimated from the lower (dilation) branch of figure $14(c)$; the compaction branch data $\left(p_{c}\right.$ and $\left.\partial v_{p, z} / \partial z\right)$ from the outer lobe were subsequently used to estimate $\kappa_{s}$. Using these bulk viscosity estimates and the compaction rate at different locations in the shallow hole, we calculated what the average collisional normal stress at different locations in this wave must be; these results are shown in figure $14(c)$ as plus signs. Although not quantitative, these estimates are close to the actual average normal stress in the shallow hole, lending support to the argument that the bulk viscosity contribution to the particle phase 


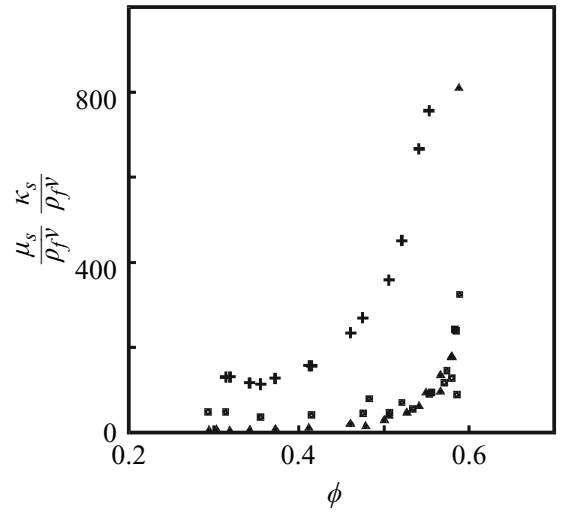

FiguRE 16. Particle phase viscosity as a function of particle volume fraction for $\bar{\phi}=0.488$. The squares (triangles) denote the shear viscosity in the compaction (dilation) branch. The plus signs are the bulk viscosity estimates in the deep void.

stress is significant. The bulk viscosity estimates corresponding to figure $14(c)$ are shown in figure 16. Also shown in this figure are the collisional shear viscosities extracted from this simulation (according to $\tau_{z z}=p_{c}-\sigma_{z z}=(4 / 3) \mu_{s} \partial v_{p, z} / \partial z$ where $\tau_{z z}$ is the deviatoric $z z$-collisional stress). It is clear that the bulk viscosity estimate is appreciably larger than the shear viscosity, which is contrary to what every theory proposed to date predicts.

One can readily speculate, at least with hindsight, as to why large departures from extant theories may arise in these flows. The fluctuating velocities in these flows are of the same order of magnitude as the local mean slip velocity. As appreciable gradients in local mean slip velocity arise over length scales of the order of only a few particle diameters, the rate of compaction is not much smaller than the rate at which the particle microstructure readjusts, and hence the magnitude of the radial distribution function at contact (which enters in the theories) is not slaved to the local mean particle volume fraction. This should be probed in future studies by studying microstructure evolution and collision statistics in these waves. The need to displace the fluid to achieve compaction and dilation of particle assemblies can also interfere with the microstructure evolution and further modify the magnitudes of the stresses.

The observations regarding momentum transfer are not very sensitive to the lateral system size (as was the case for the drag force). Figure 17 shows the collisional pressure and the bulk viscosity as a function of the solids volume fraction for the three simulations with $\bar{\phi}=0.505$ and $4 d_{p}, 5 d_{p}$ and $6 d_{p}$ as the lateral system size. The path dependence of the collisional pressure is maintained when the lateral size is reduced. Also the bulk viscosity estimates are not systematically affected.

In order to see if particle pressure $p_{s}$ (as defined in (14)) and solids phase bulk viscosity can be represented by simple functions of solids volume fraction and granular temperature, we combined the information contained in the simulations with $\bar{\phi}=0.580,0.505$ and 0.488 (all having lateral system size $6 d_{p}$ ) in figure 18 . We see that if the pressure is scaled with the granular temperature, and if the viscosity is scaled with the square root of the granular temperature, they are fairly unique functions of the solids volume fraction. Since the granular temperature is not a unique function of the solids volume fraction, this is far less the case (as also shown in figure 18) if we do not scale with granular temperature. 

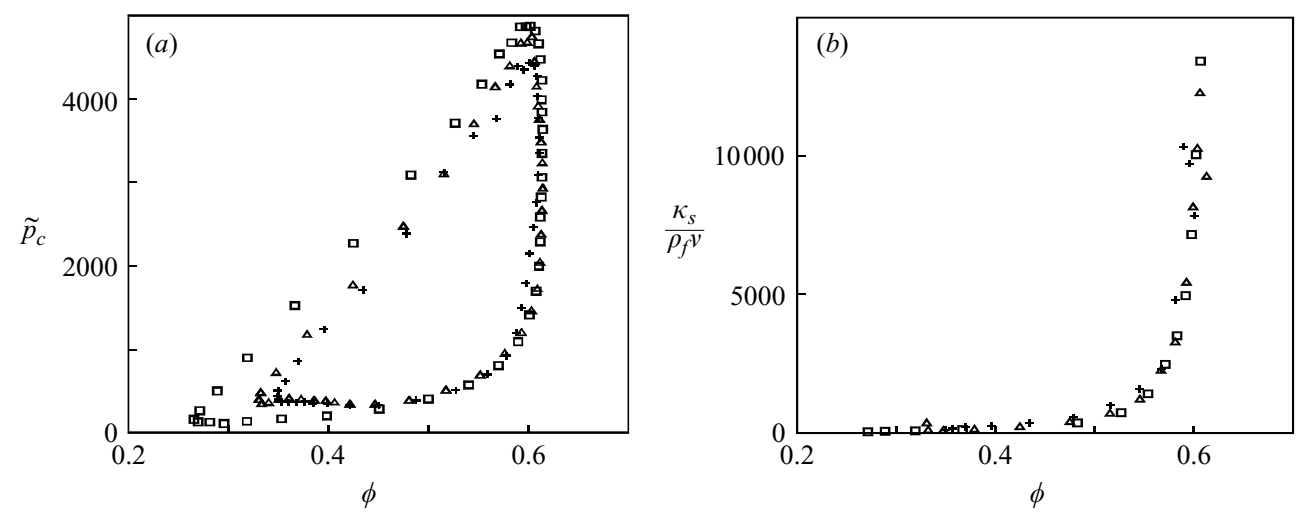

Figure 17. (a) Dimensionless collisional pressure $\tilde{p}_{c}$ and $(b)$ solids bulk viscosity $\kappa_{s}$ as a function of solids volume fraction for $\bar{\phi}=0.505$. Effect of lateral system size: squares: $6 d_{p}$; triangles: $5 d_{p}$; plus signs: $4 d_{p}$.
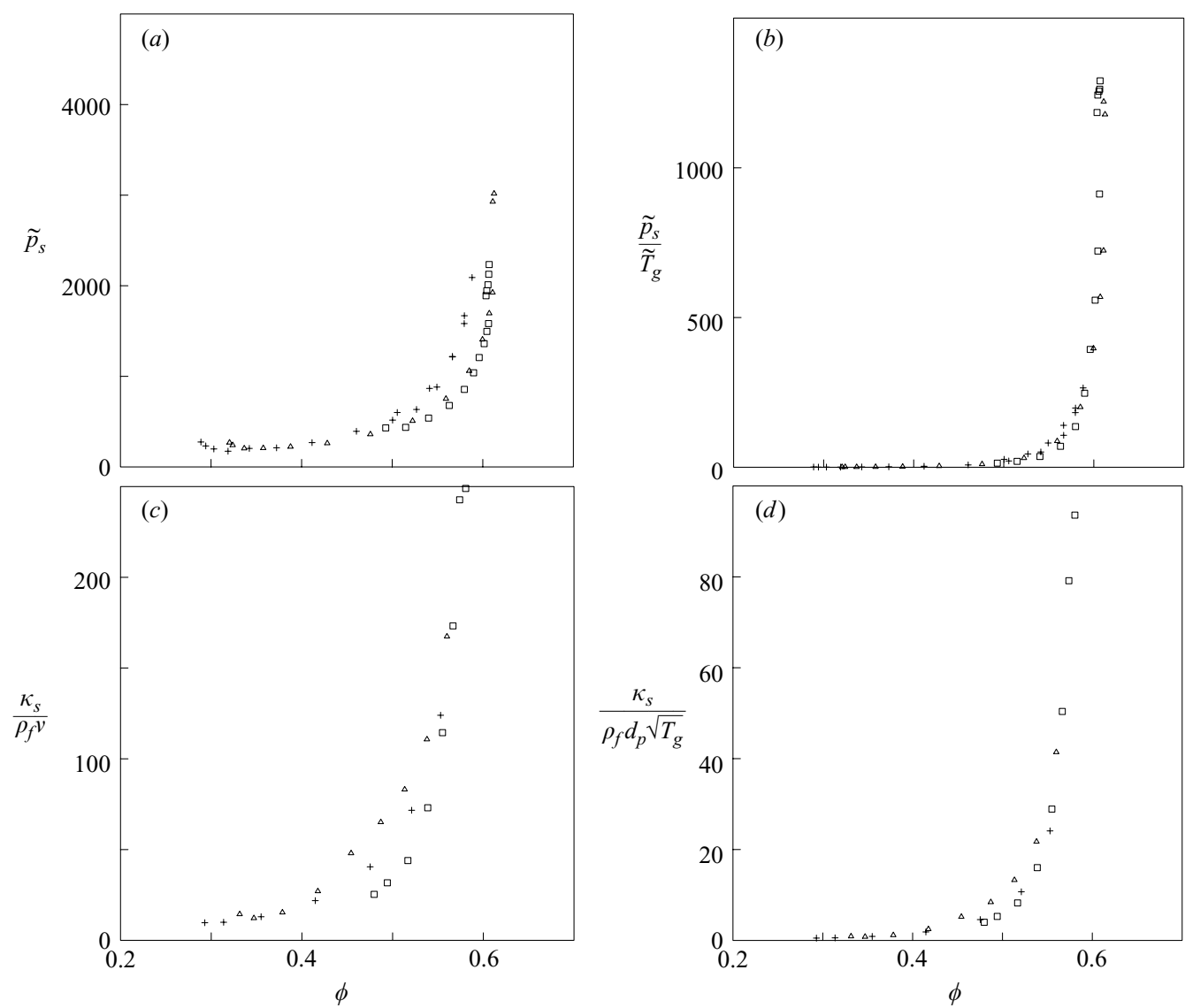

FIGURE 18. (a) Dimensionless solids phase pressure $\tilde{p}_{s}$ (as defined in equation (14)); $(b)$ solids pressure scaled with granular temperature $T_{g}$ (made dimensionless as in figure 14); $(c)$ solids phase bulk viscosity; $(d)$ solids phase bulk viscosity scaled with $\sqrt{T_{g}}$ all as a function of $\phi$ for $\bar{\phi}=0.580$ (squares), $\bar{\phi}=0.505$ (triangles), $\bar{\phi}=0.488$ (plus signs). 
If an additional variable such as the granular temperature is needed to close the continuum equations, then a transport equation for this variable, such as the kinetic theory model (Koch \& Sangani 1999) must be included in the continuum analysis. Such a transport equation includes a number of coefficients (e.g. see Koch \& Sangani 1999; Wang \& Ge 2005) and the present computations should be extended to measure these coefficients as well.

\subsection{Comparison with Duru et al.'s constitutive relations}

Duru et al. (2002) analysed their experimental findings (in terms of voidage wave shapes) in the context of the two-phase flow model equations as proposed by Anderson et al. (1995). In one-dimensional form, the model allows for planar wave solutions. Rewriting the wave solutions leads to expressions for the solids phase viscosity $\mu_{t}$ and solids phase pressure derivative $\mathrm{d} p_{s} / \mathrm{d} \phi$ in terms of parameters describing the (measured) wave shape. Their findings were presented earlier in (9). (See equations (5.1) and (6.2) in Duru et al.; we use the symbol $\mu_{t}$ instead of $\mu_{s}$ since it is a 'total' viscosity and not the shear viscosity $\mu_{s}$ as introduced in (8)). Duru et al. (2002) estimated that $\phi_{r l p} \approx \phi_{c}+0.025$ with $\phi_{c}$ being the critical solids volume fraction, i.e. the solids volume fraction at which wave instabilities set in. For the particle-fluid combination 7 (in the taxonomy by Duru et al.) that was used in our simulations, $\phi_{c}=0.543$. It is unfortunate that Duru et al. report that specifically for this combination the scaling as proposed above does not work very well. They remark that this combination hints at a $\bar{\phi}$ dependency of the solids phase viscosity.

Here, we check whether our findings in terms of solids pressure gradient and viscosity based on numerical simulations were in the same range as estimated by the experiments. We first introduce the solids phase stress as the sum of the collisional pressure and the particle streaming pressure:

$$
\sigma_{s}=\frac{1}{3}\left(\sigma_{c, x x}+\sigma_{c, y y}+\sigma_{c, z z}+\sigma_{p s, x x}+\sigma_{p s, y y}+\sigma_{p s, z z}\right) .
$$

The solids phase total viscosity $\mu_{t}$ we determine according to the one-dimensional equivalent of (8):

$$
\sigma_{s, z z}-p_{s}=-\kappa_{s} \frac{\mathrm{d} u_{p, z}}{\mathrm{~d} z}-\mu_{s} \frac{4}{3} \frac{\mathrm{d} u_{p, z}}{\mathrm{~d} z} \equiv-\mu_{t} \frac{4}{3} \frac{\mathrm{d} u_{p, z}}{\mathrm{~d} z} .
$$

In figure 19 , we show the results for the cases with $\bar{\phi}=0.505$ and $\bar{\phi}=0.580$. First, we determine the solids-phase stress as a function of $\phi$. As we did for determining the bulk viscosity, the solids phase pressure is set equal to the stress in the dilation branches of figures 19(a) and 19(b) (and the pressure derivatives are the slopes of the branches). The left-hand side of (16) is also readily determined from figures 19(a) and $19(b)$. It is the vertical distance between $\sigma_{s, z z}$ in the compaction branch, and $\sigma_{s, z z}$ (or $p_{s}$ ) in the dilation branch. Since we also know the particle velocity gradient from the simulations, $\mu_{t}$ can be calculated. The results show that our estimates are in the same range as those of Duru et al. (2002). The underestimation of the viscosity could, at least partly, be explained by the absence of bounding walls in the simulations.

\subsection{Two-dimensional waves}

In the discussion so far, the limited domain size in the lateral directions $\left(6 d_{p}\right)$ did not permit the development of two- or three-dimensional structures. This way we were able to resolve the planar waves as measured by Duru et al. (2002) in narrow tubes. Extending one (or both) lateral dimensions of the fluidized bed will lead to two- (or three-) dimensional instabilities. Two-dimensional waves and the onset of 

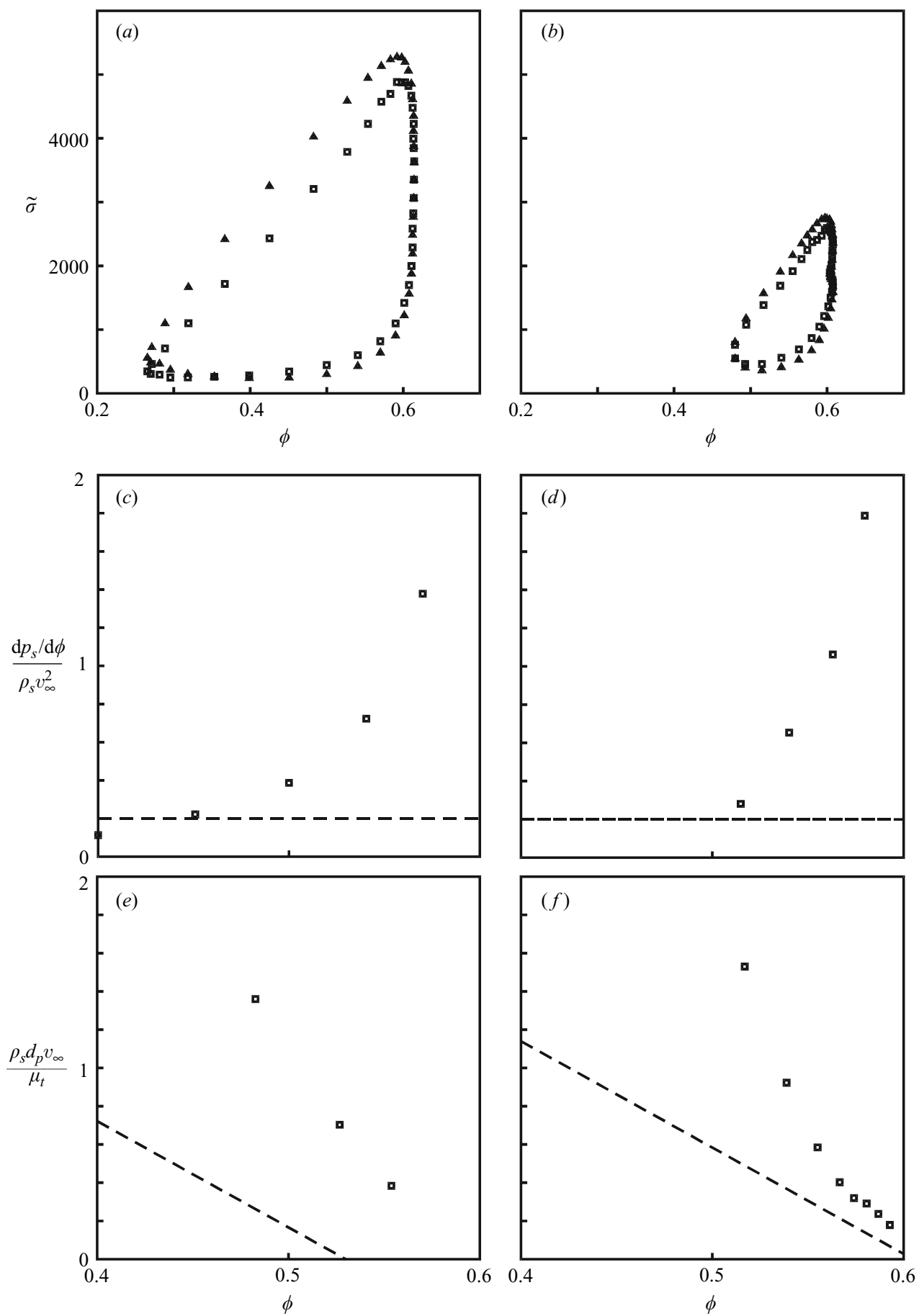

FIGURE 19. Left-hand column: $\bar{\phi}=0.505$, right-hand column: $\bar{\phi}=0.580 .(a, b)$ Average particle-phase normal stress $\tilde{\sigma}_{p}=\left(\tilde{\sigma}_{c, x x}+\tilde{\sigma}_{c, y y}+\tilde{\sigma}_{c, z z}+\tilde{\sigma}_{p s, x x}+\tilde{\sigma}_{p s, y y}+\tilde{\sigma}_{p s, z z}\right) / 3$ (squares) and the $z z$-component of the particle-phase stress $\tilde{\sigma}_{p, z z}=\tilde{\sigma}_{c, z z}+\tilde{\sigma}_{p s, z z}$ (triangles). $(c, d)$ Solids-phase pressure gradient. $(e, f)$ Inverse solids phase viscosity. The dashed lines are scaling rules as presented by Duru et al. (2002), see equation (9). 


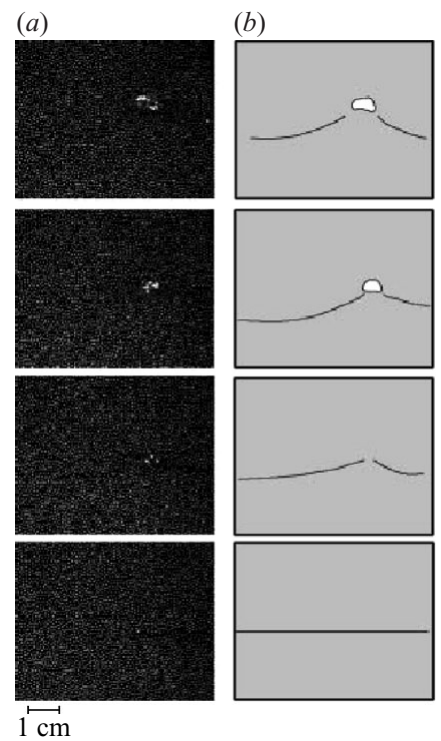

FIGURE 20. Experiment showing the onset of a bubble in a flat fluidized bed. (a) Snapshots of the bed; $(b)$ schematization of the observations. [Reprinted from Duru \& Guazelli 2002.]

bubbles have been studied experimentally by Duru \& Guazzelli (2002). They used flat liquid-fluidized beds that could not develop three-dimensional structures, and allowed for good visible observations, well-resolved void fraction measurements, and particle-tracking velocimetry measurements. Figure 20 shows a typical result of their experiments: the development of a bubble-like void, starting from a planar wave instability. In this case, steel beads (density $7.8 \times 10^{3} \mathrm{~kg} \mathrm{~m}^{-3}$ ) with a diameter of $d_{p}=1 \mathrm{~mm}$ were fluidized with water in a domain that was $120 d_{p}$ wide, $12 d_{p}$ thick and some $2000 d_{p}$ high.

Such two-dimensional structures can be simulated using the approach described in our study, provided one accepts a limited spatial resolution to keep the simulation times manageable. In order to see the evolution of two-dimensional structures, we have performed simulations in a $24 d_{p} \times 6 d_{p} \times 20 d_{p}$ periodic domain (the $20 d_{p}$ being in the streamwise direction). As an initial condition for the particle positions and velocities (translational and rotational), we juxtaposed four copies of a fully developed wave of our base case (computed in a $6 d_{p} \times 6 d_{p} \times 20 d_{p}$ periodic domain). This simulation was performed at a lower resolution than that typically used in the one-dimensional wave analysis discussed earlier; specifically, the hydrodynamic diameter of the particles was set to be 12 lattice units. As discussed earlier, at this resolution, the one-dimensional wave was resolved nicely and had a speed comparable to simulations with $d_{p}=16$ and 24 lattice units. The density ratio was set to 8 . The viscosity and body force were chosen such that the terminal velocity of a single bead was 0.04 (in lattice units) and the Reynolds number based on the terminal velocity matched the value in the experiments $(R e=400)$. The collisions were smooth and elastic $(\mu=0, e=1)$.

Figure 21 shows how the numerical system developed a bubble very similar to the experimental observations: the initially plane wave buckles and, at its crest, forms a bubble-like void. The particle velocity field in the vicinity shows qualitative similarity with the field measured by Duru \& Guazzelli (2002) (see figure 22). We again note that non-ideality of bead-bead collisions is not an essential condition for resolving 

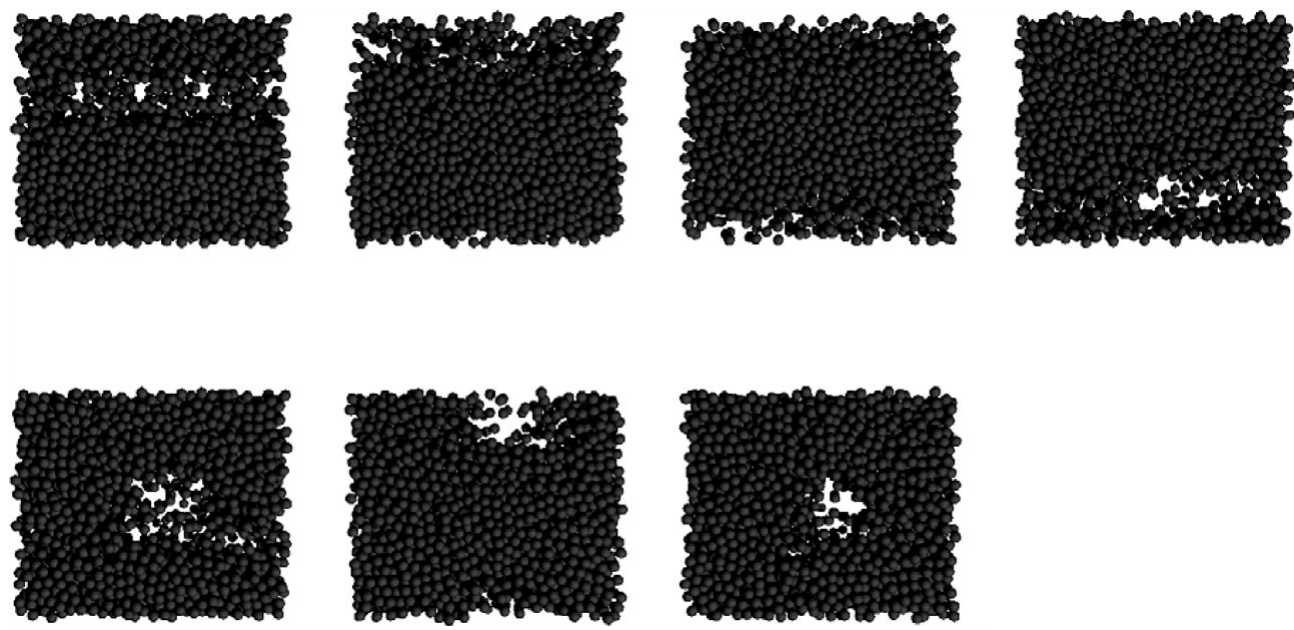

FIGURE 21. Series of snapshots showing bubble formation. The upper left frame shows the initial condition. The time-spacing between the subsequent (left-to-right, top-to-bottom) frames is $0.057 d_{p}^{2} / \nu$.

(a)

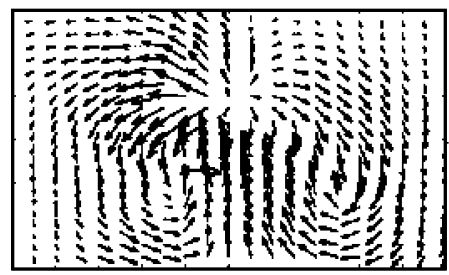

(b)

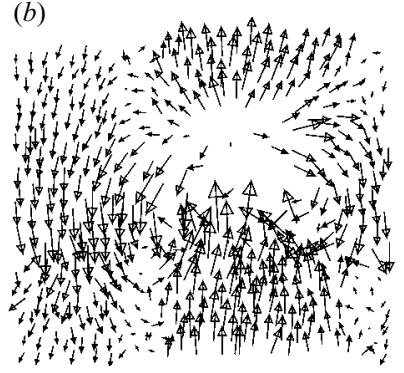

Figure 22. (a) Measured [reprinted from Duru \& Guazelli 2002], and (b) simulated particle velocities in the vicinity of a bubble.

the behaviour of void fraction instabilities in fluidized beds. The domain size in the flow direction is too short for the bubble to behave as an isolated bubble. The periodic system resembles a bubble train, which generally has a higher velocity than an isolated bubble. If we estimate the bubble rise velocity and translate it back to the experimental steel-water system, the bubble rise velocity in the simulation is approximately $15 \mathrm{~cm} \mathrm{~s}^{-1}$. The bubble radius is approximately $0.4 \mathrm{~cm}$. This bubble size and rise velocity combination is at the lower end of the range of rise velocities for bubble trains which is 15 to $22 \mathrm{~cm} \mathrm{~s}^{-1}$ according to the experiments (Duru \& Guazzelli 2002, their figure 15b). These results on the evolution of two-dimensional structures serve to validate the numerical simulation approach followed in this study to generate the computational data on one-dimensional travelling waves. More in-depth analysis of the results of such multi-dimensional structures will be addressed in a future paper.

\section{Summary}

Flow of dense liquid-fluidized suspensions of monodisperse spheres has been simulated in detail by means of the lattice-Boltzmann method and a hard-sphere approach for direct particle-particle interactions. Lubrication forces were implemented 
to represent the fluid dynamics at close proximity to solid surfaces. The formation of one-dimensional waves in narrow periodic domains and a bubble-like void in wider periodic domains observed in the simulations is in qualitative agreement with the experimental data from Duru et al. (2002) and Duru \& Guazzelli (2002). The onset, shape and speed of the one-dimensional planar waves were largely insensitive to the parameters governing the hard-sphere collisions (namely, the restitution and friction coefficients). The wave speeds extracted from the simulations were comparable to the reported experimental values. These results served as qualitative validation of the simulation tool and justified more in-depth analysis of other results extracted from the simulations (which are not experimentally accessible).

By ensemble-averaging the simulation data in a frame of reference moving with a fully developed wave, we determined the spatial variation of various local-average quantities typically appearing in two-fluid models for such flows. The presence of a void, travelling as a wave in the direction opposite to gravity, allowed us to assess the role of inhomogeneities and the sensitivity of the forces and stresses to solids volume fraction in a single simulation.

The fluid-particle interaction (drag) force data from each simulation could be summarized in a form reminiscent of the Richardson \& Zaki model,

$$
f_{f p, h y d}=\frac{g}{v_{o}}\left(\rho_{s}-\rho_{f}\right) \frac{\phi}{(1-\phi)^{N-2}}\left(v_{f}-v_{p}\right) .
$$

The exponent $N$ is close to the value obtained by Richardson \& Zaki (1954). In the standard Richardson-Zaki formulation (Batchelor 1988; Jackson 2000), the velocity $v_{o}$ appearing in the above equation is the terminal settling velocity of an isolated particle, $v_{\infty}$. Our results for $v_{o}$ were very sensitive for the size of the flow domain. Extrapolation to infinitely sized domains, however, showed that $v_{o}$ approached $v_{\infty}$.

The simulations allowed for detailed evaluation of the relative importance of the momentum transfer mechanisms. In the dense regions of the system, momentum transfer occurs predominantly through collisions. In the void, fluid and particle streaming are of comparable importance. Stresses due to lubrication, and the fluidphase viscous stresses were of minor importance.

The travelling wave includes regions where the particle assembly undergoes compaction (just below the rising void) and dilation (just above the void). A remarkable degree of asymmetry was observed in the stresses in these two regions. The average collisional normal stress was appreciably larger in the compaction branch and it is shown that it depended on the rate of compaction. In contrast, the effect of the dilation rate on the average collisional normal stress was only small. These results are consistent with the notion of a bulk viscosity which assumed different values in the dilation and compaction branches.

At a more fundamental level, the dependence of the average collisional normal stress on the rate of compaction is probably a consequence of the non-equilibrium microstructure of the particle assembly, which, in turn, derives from the fact that the rates of compaction and dilation of the assembly in these waves are not slow compared to the rate at which the microstructure relaxes. These observations suggest that higher- order closures which recognize the slow evolution of the microstructure in these flows and account for the effects of non-equilibrium microstructure on the drag and the stresses may be required; such closures, however, are difficult to develop, and it is likely that we will continue to rely on simple models which capture the gross effects, but not all the details. Nevertheless, analysis of the type described here exposes the limitations of such models. 
This work was partly supported by grants from the American Chemical Society Petroleum Research Fund, the ExxonMobil Research \& Engineering Company and the Delft Center for Sustainable Industrial Processes.

\section{Appendix A. Effective equations of motion for the particles in the presence of internal fluid}

In our simulations, the fluid and the particles experience effective body forces which can be related to the acceleration due to gravity, $\boldsymbol{g}=-\boldsymbol{g} \boldsymbol{e}_{z}$, as follows. The net gravity force acting on each spherical particle is

$$
\boldsymbol{F}_{G}=-\left(\rho_{s}-\bar{\rho}\right) \frac{\pi}{6} d_{p}^{3} g \boldsymbol{e}_{z}
$$

and the force per unit volume acting on the (internal and external) fluid is

$$
\boldsymbol{f}_{B}=\left(\bar{\rho}-\rho_{f}\right) g \boldsymbol{e}_{z},
$$

with $\bar{\rho}=\bar{\phi} \rho_{s}+(1-\bar{\phi}) \rho_{f}$ the domain-average density of the fluid-solid mixture. It can readily be recognized that the imposed body force accounts for both the body force due to gravity acting on the fluid and the particles, and the contribution of the average pressure gradient to the travelling waves in physical experiments.

The fluid inside the spherical particles is an artefact of the forcing scheme. As long as the density of the solid is higher than the density of the fluid, the effects of the internal fluid can be effectively corrected for. The force $\boldsymbol{F}_{L B}$ acting on the fluid determined by the forcing method is the sum of the force required to accelerate the internal fluid and the force of the particle acting on the external fluid. Since the internal fluid behaves largely as a solid body and it has nearly the same linear velocity of the sphere, we can partition $\boldsymbol{F}_{L B}$ as follows: $\boldsymbol{F}_{L B}=\boldsymbol{F}_{L B, 1}+\boldsymbol{F}_{\text {ext }}$. where the force $\boldsymbol{F}_{L B, 1}$ is the component that ensures that the internal fluid translates with the particle; $\boldsymbol{F}_{\text {ext }}$ is the force on the external fluid owing to the particle. The overall linear momentum balance for the internal fluid can be written as

$$
\rho_{f} \frac{\pi}{6} \mathrm{~d}_{p}^{3} \frac{\mathrm{d} \boldsymbol{v}_{p}}{\mathrm{~d} t}=+\boldsymbol{F}_{L B, 1}+\left(\bar{\rho}-\rho_{f}\right) \frac{\pi}{6} d_{p}^{3} g \boldsymbol{e}_{z}=\boldsymbol{F}_{L B}-\boldsymbol{F}_{e x t}+\left(\bar{\rho}-\rho_{f}\right) \frac{\pi}{6} d_{p}^{3} g \boldsymbol{e}_{z},
$$

where it has been recognized that the internal fluid translates with the particle. The corresponding equation for the particle is then

$$
\rho_{s} \frac{\pi}{6} d_{p}^{3} \frac{\mathrm{d} \boldsymbol{v}_{p}}{\mathrm{~d} t}=-\boldsymbol{F}_{\text {ext }}-\left(\rho_{s}-\bar{\rho}\right) \frac{\pi}{6} d_{p}^{3} g \boldsymbol{e}_{z} .
$$

Lubrication forces which arise because of inadequate resolution of the flow in between neighbouring particles and those arising from direct particle-particle interactions (e.g. collisions) can readily be added to the right-hand side of this equation.

Upon combining these equations, we obtain

$$
\left(\rho_{s}-\rho_{f}\right) \frac{\pi}{6} d_{p}^{3} \frac{\mathrm{d} \boldsymbol{v}_{p}}{\mathrm{~d} t}=-\boldsymbol{F}_{L B}-\left(\rho_{s}-\rho_{f}\right) \frac{\pi}{6} d_{p}^{3} g \boldsymbol{e}_{z} .
$$

Following the same reasoning, we obtain the following angular momentum balance:

$$
\left(\rho_{s}-\rho_{f}\right) \frac{\pi}{60} d_{p}^{5} \frac{\mathrm{d} \boldsymbol{\Omega}_{p}}{\mathrm{~d} t}=-\boldsymbol{T}_{L B}
$$

where $\boldsymbol{T}_{L B}$ is the torque acting on the fluid determined by the forcing method. 


\section{Appendix B. Lubrication forces}

The general framework for lubrication forces and torques acting on two particles ( 1 and 2$)$ as a result of the relative motion of their surfaces can be written in the form of the following vector equation:

$$
\left[\begin{array}{l}
\boldsymbol{F}_{l u b, 1} \\
\boldsymbol{T}_{l u b, 1} \\
\boldsymbol{T}_{l u b, 2}
\end{array}\right]=\left[\begin{array}{lll}
\boldsymbol{A}_{11} & -\boldsymbol{B}_{11} & \boldsymbol{B}_{22} \\
\boldsymbol{B}_{11} & \boldsymbol{C}_{11} & \boldsymbol{C}_{12} \\
-\boldsymbol{B}_{22} & \boldsymbol{C}_{12} & \boldsymbol{C}_{22}
\end{array}\right]\left[\begin{array}{c}
\boldsymbol{v}_{p, 12} \\
\boldsymbol{\Omega}_{1} \\
\boldsymbol{\Omega}_{2}
\end{array}\right]
$$

with $\boldsymbol{F}_{l u b, 2}=-\boldsymbol{F}_{l u b, 1}$ and $\boldsymbol{v}_{p, 12}=\boldsymbol{v}_{p, 1}-\boldsymbol{v}_{p, 2}$ (Kim \& Karila 1991; Nguyen \& Ladd 2002). In the tensors $\boldsymbol{A}_{11}, \boldsymbol{B}_{11}, \boldsymbol{B}_{22}, \boldsymbol{C}_{11}, \boldsymbol{C}_{22}$, and $\boldsymbol{C}_{12}$, we only use the leading-order terms in the parameter $d_{p} / h$, with $h$ the minimum spacing of the particle surfaces. For the radial lubrication force (contained in the diagonal elements of the $\boldsymbol{A}_{11}$ matrix in the equation $\boldsymbol{F}_{l u b, 1}=\boldsymbol{A}_{11} \boldsymbol{v}_{p, 12}$,) the leading order is $d_{p} / h$, while for the tangential lubrication forces and torques it is $\ln \left(d_{p} / h\right)$ (Kim \& Karila 1991). Two modifications to the above expressions were implemented to tailor them to our numerical needs:

(i) Lubrication only acts if particle separation is less than $\delta=0.1 d_{p}$ (which is equivalent to 1.6 lattice spacing in the default resolution). To switch on/off the lubrication force smoothly at $h=\delta$, in the lubrication expressions, $d_{p} / h$ is replaced by $d_{p} / h-d_{p} / \delta$, and $\ln \left(d_{p} / h\right)$ by $\ln \left(d_{p} / h\right)-\ln \left(d_{p} / \delta\right)$ (Nguyen $\&$ Ladd 2002).

(ii) The lubrication force saturates once the particles are very close $\left(\right.$ at $\left.10^{-4} d_{p}\right)$. The latter restriction we use for numerical reasons (to avoid high force levels and associated instabilities) but also with the surface roughness of the particles and/or the mean free path of the fluid in mind.

By comparing the behaviour of a (translating and spinning) spherical particle in the vicinity of a flat wall, Nguyen \& Ladd (2002) validated the way they connected lubrication forces (radial and tangential) and torques to the lattice-Boltzmann method. The analytical solutions for these test cases are due to Cichocki \& Jones (1998).

We have also repeated the test simulation presented by Verberg \& Koch (2006) namely, the normal approach of a spherical particle towards a flat surface. The solid line in figure 23 represents the analytical solution for the force experienced by the particle in Stokes flow. The open squares indicate the fluid-particle interaction force computed by the LBM and the open triangles result when the lubrication force is added to the fluid-particle interaction force computed in the simulations. In this simulation, the resolution of the sphere was $a=16$; by increasing the resolution, the accuracy can be improved further.

Experiments and modelling by Joseph et al. (2001) indicate that the radial lubrication force can capture qualitatively the effect of the Stokes number on the effective coefficient of restitution $e_{\text {eff }}$ of a submerged sphere impacting on a flat wall. If we compare our procedure for applying the lubrication force as sketched above to the model of Joseph et al. for estimating $e_{\text {effi, }}$ they differ in what happens at close proximity to the lubricating surfaces, and in that we have included tangential lubrication, the latter being of negligible significance. Joseph et al. let the surfaces collide at a critical distance (commensurate with the surface roughness) away from the point corresponding to perfectly smooth surfaces whereas we saturate the lubrication force at a distance at which we expect roughness to become an important factor and subsequently let the smooth surfaces collide. This implies that in our model, the particle slows down slightly more and that $e_{\text {eff }}$ will become slightly smaller. While 


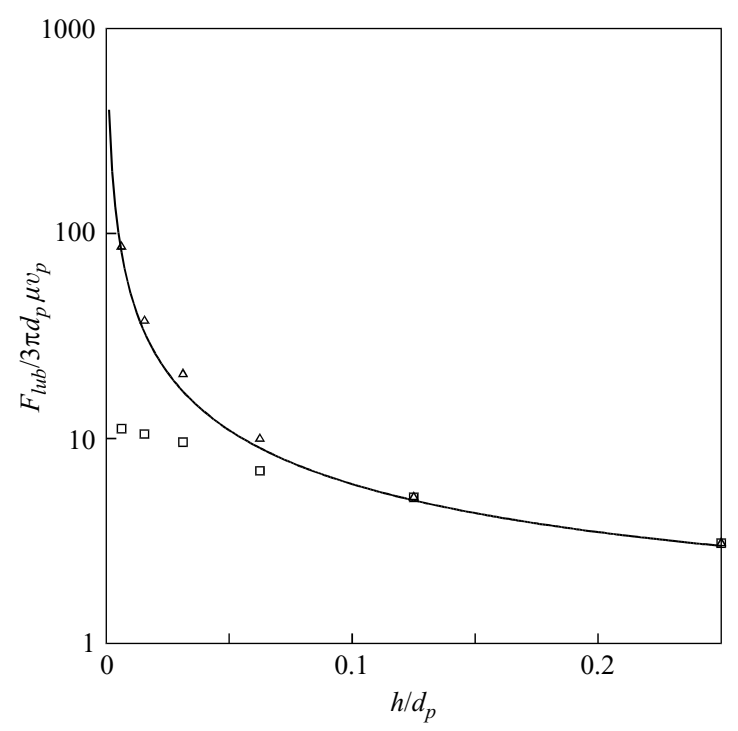

FIGURE 23. Force on a spherical particle approaching a flat wall with normal velocity $v_{p}$, as a function of the spacing $h$. Line: analytical solution (Cichocki \& Jones 1998); squares: force as resolved by the LBM; triangles: force resolved by LBM plus subgrid scale lubrication force.

travelling from the critical distance to contact, and back, i.e. at constant (saturated) lubrication force, the particle velocity will decay exponentially. The factor with which the particle velocity has decayed after having travelled the critical distance twice is approximately $\exp (-2 / S t)$ with the Stokes number based on the approach velocity $u_{0}: S t=(1 / 9)\left(\rho_{s} / \rho_{f}\right)\left(d_{p} u_{0} / v\right)$. This factor does not depend on the critical distance. It would only slightly alter the modelling results as confronted with experimental data in figure 12 of Joseph et al. (2001): the critical Stokes number would be shifted to slightly higher values, and the effective restitution coefficient would reduce slightly (at $S t=10^{2}$ with a factor 0.98). The scatter in the experimental data of Joseph et al. does not allow for assessing these subtle differences. In sum, the way we handle lubrication in the present paper differs only slightly from the scheme described by Joseph et al. (2001) and the difference is well within the scatter of their experimental data.

Short-range lubrication forces are similar for particle-particle and particle-wall interactions (see also Yang \& Hunt 2006). The same mathematical approach can be used to capture the influence of lubrication force on the effective coefficient of restitution for collisions between submerged particles and between submerged particles and wall (Joseph et al. 2001; Gondret, Lance \& Petit 2002; Cichocki \& Jones 1998). Thus, it follows that the implementation of the lubrication force in our simulations is also consistent with experimental results on submerged particle-particle collisions.

\section{Appendix C. Procedure to determine various local-average quantities along the wave}

In the co-travelling frame, the flow domain was divided into many slices of equal thickness. For example, in the base case having a wavelength of $20 d_{p}$, we employed 40 slices so that each slice had a thickness of $0.5 d_{p}$. The particle volume fraction in 

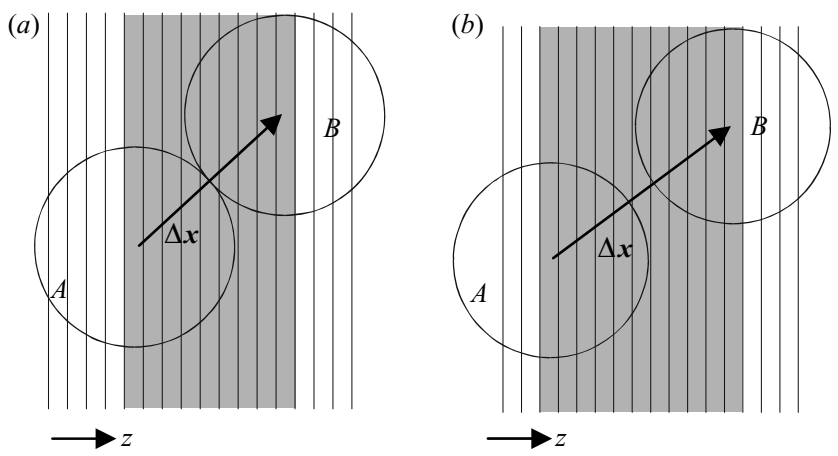

Figure 24. Definitions as used in determining $(a)$ the collisional stress, $(b)$ the stresses due to lubrication.

each slice was determined by dividing the volume occupied by solid material over the total slice volume.

The fluid velocity was averaged over the slice by considering the external fluid (LB) nodes only: $\left\langle\boldsymbol{v}_{f}\right\rangle=\sum \boldsymbol{v}_{f, i} V_{i} / V_{f}$ with the sum over the fluid nodes $i$ in the slice. $V_{i}$ is the node volume, and $V_{f}$ is the total fluid volume in the slice.

For the ( $\alpha$ th component of the) mean-square fluid velocity a similar procedure applies: $\left\langle v_{f \alpha}^{2}\right\rangle=\sum v_{f \alpha, i}^{2} V_{i} / V_{f}$. The root-mean-square value of the fluid velocity fluctuations, $v_{f \alpha}^{\prime}$, is then given by $v_{f \alpha}^{\prime}=\sqrt{\left\langle v_{f \alpha}^{2}\right\rangle-\left\langle v_{f \alpha}\right\rangle^{2}}$. To find the normal components of the fluid streaming stress, we averaged over the total slice volume: $\sigma_{f s, \alpha \alpha}=\rho_{f}\left(v_{f \alpha}^{\prime}\right)^{2} V_{f} / V$. Here, $V$ denotes the total volume of the slice.

The fluid-to-particle force acting on each particle was first found by summing the forces that are imposed to satisfy the no-slip condition at the sphere surface, and these forces were localized at the particle centre. The total fluid-to-particle force in a slice was then found as: $\left\langle\boldsymbol{F}_{f \rightarrow p}\right\rangle=-\sum \boldsymbol{F}_{i} V_{i} / V$, where we sum over all the particles whose centres lie in the slice. The negative sign is included as $\boldsymbol{F}_{i}$ is the force on the fluid due to the particle.

The mean particle velocity was determined by averaging the velocities of the particles having their centres in the slice under consideration. The same procedure is applied to find the mean-square particle velocity. From the mean-square and mean velocities we determine the (root) mean-square of the velocity fluctuations. The normal particle streaming stresses are then:

$$
\sigma_{p s, \alpha \alpha}=\frac{\rho_{s} \phi\left(v_{s \alpha}^{\prime}\right)^{2}}{V} .
$$

Finding the collisional stress involved many steps. Consider a binary collision as sketched in figure 24. The circles denote the two spheres $A$ and $B$ that collide and the vertical lines are the LB grid planes normal to $z$ (which is the direction in which the wave travels). Suppose at the collision the particle velocities change as

$$
\boldsymbol{v}_{A 1}=\boldsymbol{v}_{A 0}+\boldsymbol{a} \quad \boldsymbol{v}_{B 1}=\boldsymbol{v}_{B 0}-\boldsymbol{a}
$$

(index 0: before collision; index 1: after collision). Then an amount of $z$-momentum equal to $m_{p} a_{z}$ is transferred in the $z$-direction over a distance $\Delta z, x$-momentum $m_{p} a_{x}$ in the $x$-direction over $\Delta x$, and $y$-momentum $m_{p} a_{y}$ in the $y$-direction over $\Delta y$ (with $m_{p}$ the mass of the sphere, and $\Delta x, \Delta y$, and $\Delta z$ the components of the vector $\Delta \boldsymbol{x}$ separating the sphere centres, see figure 24(a). We evenly distribute this 
momentum transfer $m_{p} a_{z} \Delta z, m_{p} a_{x} \Delta x, m_{p} a_{y} \Delta y$ over the grey LB volume (i.e. the volume in between the centres of the two spheres involved). The contributions of various collisions in a time interval $\Delta t$ are summed up to determine the collisional stress:

$$
\sigma_{c, \alpha \alpha, k}=\frac{1}{\Delta t} \sum \frac{m_{p} a_{\alpha} \Delta \alpha}{V},
$$

with $\alpha=x, y$ or $z$, and where the sum is over all collisions in the time interval with the axial $(z)$ location $k$ lying in between the sphere centres at collision. $V$ denotes the volume of the domain shown in grey in figure 24(a). This procedure gave us a stress profile with the spatial resolution of the LB simulation. By averaging over many realizations, we were able to obtain smooth results at the slice resolution that we have used for the other statistical quantities described above.

The procedure to compute the lubrication stress is analogous to the collisional stress. The momentum exchange between two spheres is now not instantaneous (as it is in a hard-sphere collision), but acts over a time interval $\delta t$ (in the text, this time interval is referred to as a sub-time-step), and the particles are not in contact. The geometrical picture (see figure $24(b)$ ) is similar. In the equation for the lubrication stress, $m_{p} a_{\alpha}$ is replaced by $F_{L \alpha} \delta t$ with $F_{L \alpha}$ the $\alpha$ th component of the lubrication force:

$$
\sigma_{L, \alpha \alpha, k}=\frac{1}{\Delta t} \sum_{i} \frac{F_{L \alpha} \delta t_{i} \Delta \alpha}{V},
$$

and the time interval $\Delta t=\sum_{i} \delta t_{i}$. The volume $V$ is now the grey volume in figure 24(b).

\section{Appendix D. Evaluation of the fluid-particle interaction force}

The spatial averaging procedure leading to the averaged equations of motion uses a normalized weighting function $g$, centred at location $\boldsymbol{x}$ :

$$
\int_{V} \boldsymbol{g}(|\boldsymbol{x}-\boldsymbol{y}|) \mathrm{d} V_{y}=1 .
$$

Here, $V$ denotes the entire region occupied by the fluid-particle mixture. The local fluid volume fraction is given by

$$
(1-\phi)(\boldsymbol{x})=\left\langle\int_{V_{f}} g(|\boldsymbol{x}-\boldsymbol{y}|) \mathrm{d} V_{y}\right\rangle .
$$

Here $V_{f}$ denotes the region occupied the fluid, $\phi$ is the local particle volume fraction, and the angle brackets indicates averaging over many realizations. The local average fluid velocity, $\boldsymbol{u}_{f}(\boldsymbol{x})$, is given by

$$
\boldsymbol{u}_{f}(\boldsymbol{x})=\frac{1}{1-\phi}\left\langle\int_{V_{f}} \boldsymbol{u}(\boldsymbol{y}) g(|\boldsymbol{x}-\boldsymbol{y}|) \mathrm{d} V_{y}\right\rangle,
$$

where $\boldsymbol{u}(\boldsymbol{y})$ is the actual fluid velocity at $\boldsymbol{y}$. Particle averages are defined on the basis of the location of the sphere centres, $\boldsymbol{x}_{p}$. Then the number density $n(\boldsymbol{x})$ is given by

$$
n(\boldsymbol{x})=\left\langle\sum_{P} g\left(\left|\boldsymbol{x}-\boldsymbol{x}_{p}\right|\right)\right\rangle .
$$


Consider a case where $n$ varies only in one spatial direction, $z$. Then, we can show that

$$
\phi(z)=\frac{4}{3} \pi a^{3} \mathrm{n}(z)+\frac{2}{15} \pi a^{5} \frac{\mathrm{d}^{2} \mathrm{n}}{\mathrm{d} z^{2}}+\text { h.o.t. }
$$

where now $a$ denotes particle radius. As discussed by Ten Cate $\&$ Sundaresan (2006a), in the one-dimensional travelling waves, the second (and the higher-order) terms on the right-hand side of (D 5) are negligible and

$$
\phi(z) \approx \frac{4}{3} \pi a^{3} \mathrm{n}(z)
$$

The local average particle velocity $\boldsymbol{u}_{s}(\boldsymbol{x})$ is given by

$$
\boldsymbol{u}_{s}(\boldsymbol{x})=\frac{1}{n(\boldsymbol{x})}\left\langle\sum_{P} \boldsymbol{u}_{p} g\left(\left|\boldsymbol{x}-\boldsymbol{x}_{p}\right|\right)\right\rangle
$$

where $\boldsymbol{u}_{p}$ is the velocity of the $p$ th particle located at $\boldsymbol{x}_{p}$.

The two-fluid model that results from the averaging process takes the following form:

$$
\begin{gathered}
\frac{\partial \phi}{\partial t}+\nabla \cdot\left(\phi \boldsymbol{u}_{s}\right)=0 \\
\frac{\partial}{\partial t}\left[\rho_{f}(1-\phi)\right]+\nabla \cdot\left[\rho_{f}(1-\phi) \boldsymbol{u}_{f}\right]=0 \\
\rho_{f}(1-\phi)\left[\frac{\partial \boldsymbol{u}_{f}}{\partial t}+\boldsymbol{u}_{f} \cdot \nabla \boldsymbol{u}_{f}\right]=\nabla \cdot \pi_{f}-\nabla \cdot \boldsymbol{\sigma}_{f s}-\boldsymbol{F}+\rho_{f}(1-\phi) \boldsymbol{g}+\boldsymbol{T}, \\
\rho_{s} \phi\left[\frac{\partial \boldsymbol{u}_{s}}{\partial t}+\boldsymbol{u}_{s} \cdot \nabla u_{s}\right]=\nabla \cdot \boldsymbol{\sigma}_{s}+\boldsymbol{F}+\rho_{s} \phi \boldsymbol{g} .
\end{gathered}
$$

Here, $\sigma_{s}$ is the sum of collisional, lubrication and streaming stresses in the particle phase. $\pi_{f}$ is the local-average fluid-phase stress:

$$
\boldsymbol{\pi}_{f}=\frac{1}{1-\phi}\left\langle\int_{V_{f}} \boldsymbol{\sigma}(\boldsymbol{y}) g(|\boldsymbol{x}-\boldsymbol{y}|) \mathrm{d} V_{y}\right\rangle,
$$

where $\boldsymbol{\sigma}(\boldsymbol{y})$ is the local fluid-phase stress. $\boldsymbol{\sigma}_{f s}$, the fluid-phase streaming stress, is given by,

$$
\boldsymbol{\sigma}_{f s}=\left\langle\int_{v_{f}} \rho_{f}\left(\boldsymbol{u}(\boldsymbol{y})-\boldsymbol{u}_{f}(\boldsymbol{y})\right)\left(\boldsymbol{u}(\boldsymbol{y})-\boldsymbol{u}_{f}(\boldsymbol{y})\right) g(|\boldsymbol{x}-\boldsymbol{y}|) \mathrm{d} V_{y}\right\rangle .
$$

The fluid-particle interaction force, $\boldsymbol{F}$, is defined as:

$$
\boldsymbol{F}=\left\langle\sum_{p} g\left(\left|\boldsymbol{x}-\boldsymbol{x}_{p}\right|\right) \int_{s_{p}} \boldsymbol{n} \cdot \boldsymbol{\sigma}(\boldsymbol{y}) \mathrm{d} S_{y}\right\rangle .
$$

Note that, in this expression, $\int_{s_{p}} \boldsymbol{n} \cdot \boldsymbol{\sigma}(\boldsymbol{y}) \mathrm{d} S_{y}$ is the total fluid-particle force over the surface of the $p$ th particle, and it is assigned to the centre of the $p$ th particle.

The force $\boldsymbol{T}$ (per unite bed volume) is due to the finite size of the particles. The total fluid-particle force which appears in the fluid phase momentum balance is

$$
\left\langle\sum_{p} \int_{s_{p}} \boldsymbol{n} \cdot \boldsymbol{\sigma}(\boldsymbol{y}) g(|\boldsymbol{x}-\boldsymbol{y}|) \mathrm{d} S_{y}\right\rangle
$$


which is different from $\boldsymbol{F}$ given by (D 14). This difference is the vector $\boldsymbol{T}$. This force has been estimated from LB simulations of flows in inhomogeneous packed beds by Ten Cate \& Sundaresan (2006a). To leading order, we can show that

$$
T=\nabla \cdot t_{1}(x),
$$

where

$$
\boldsymbol{t}_{1}(\boldsymbol{x})=a\left\langle\sum_{p} g\left(\left|\boldsymbol{x}-\boldsymbol{x}_{p}\right|\right) \int_{s_{p}} \boldsymbol{n} \boldsymbol{n} \cdot\left(\sigma(\boldsymbol{y})-\boldsymbol{\pi}_{f}(\boldsymbol{y})\right) \mathrm{d} S_{y}\right\rangle
$$

We label $\boldsymbol{t}_{1}(\boldsymbol{x})$ as fluid-particle stress. We have evaluated $\boldsymbol{t}_{1}(\boldsymbol{x})$ from our LB simulation results and found it to be generally small; separately, Ten Cate \& Sundaresan (2006a) found $\boldsymbol{T}$ to be quite small, and so we will not consider it any further in our analysis. Equation (D 10) then simplifies to

$$
\rho_{f}(1-\phi)\left[\frac{\partial \boldsymbol{u}_{f}}{\partial t}+\boldsymbol{u}_{f} \cdot \nabla \boldsymbol{u}_{f}\right]=\nabla \cdot \pi_{f}-\nabla \cdot \boldsymbol{\sigma}_{f s}-\boldsymbol{F}+\rho_{f}(1-\phi) \boldsymbol{g} .
$$

Ten Cate \& Sundaresan (2006a) studied fluid flow in inhomogeneous packed beds, and found that $\sigma_{f s}$ was comparable to $\rho_{f}(1-\phi) \boldsymbol{u}_{f} \boldsymbol{u}_{f}$ and that contributions from both of there were much smaller than those from the other terms on the right-hand side of (D 17). In our LB simulations, we found the fluid-phase streaming stress to be measurably large in the void region, and so there is some basis retaining this term and for exploring its consequence. Combining $\pi_{f}$ and $\sigma_{f s}$ into a single effective fluid-phase stress tensor $\boldsymbol{\sigma}_{f}$, we can write (D 17) as:

$$
\rho_{f}(1-\phi)\left[\frac{\partial \boldsymbol{u}_{f}}{\partial t}+\boldsymbol{u}_{f} \cdot \nabla \boldsymbol{u}_{f}\right]=\nabla \cdot \boldsymbol{\sigma}_{f}-\boldsymbol{F}+\rho_{f}(1-\phi) \boldsymbol{g} .
$$

We now express $\boldsymbol{F}$ as a sum of two contributions (e.g. see Jackson 2000):

$$
\boldsymbol{F}=\boldsymbol{f}+\phi \nabla \cdot \boldsymbol{\sigma}_{f} .
$$

For one-dimensional flows, then the $z$ component takes the form

$$
F_{z}=f_{z}-\phi \frac{\partial p}{\partial z}+\phi \frac{\partial \tau_{f, z z}}{\partial z}-\phi \frac{\partial \sigma_{f s, z z}}{\partial z} .
$$

Here, $\tau_{f, z z}$ is the $z z$-component of the local-average fluid phase viscous stress. As the viscous stress was found to be negligibly small in our problem, we can simplify (D 20) as

$$
F_{z} \approx f_{z}-\phi \frac{\partial p}{\partial z}-\phi \frac{\partial \sigma_{f s, z z}}{\partial z} .
$$

This equation appears in the main text as (10).

\section{REFERENCES}

Agrawal, K., Loezos, P. N., Syamlal, M. \& Sundaresan, S. 2001 The role of meso-scale structures in rapid gas-solid flows. J. Fluid Mech. 445, 151-185.

Aidun, C. K., Lu, Y. \& Ding, E. J. 1998 Direct analysis of particle suspensions with inertia using the discrete Boltzmann equation. J. Fluid Mech. 373, 287-311.

Anderson, K., Sundaresan, S. \& JaCKson, R. 1995 Instabilities and the formation of bubbles in fluidized beds. J. Fluid Mech. 303, 327-366.

ANDERson, T. B. \& JACKSON, R. 1969 A fluid mechanical description of fluidized beds - comparison of theory and experiment. Indust. Engng Chem. Fund. 8, 137-144. 
Auton, T. R., Hunt, J. C. R. \& Prud'homme, M. 1988 The force exerted on a body in inviscid unsteady non-uniform rotational flow. J. Fluid Mech. 197, 241-257.

Batchelor, G. K. 1988 A new theory of the instability of a uniform fluidized bed. J. Fluid Mech. 193, 75-110.

Brady, J. F., Khair, A. S. \& Swaroop, M. 2006 On the bulk viscosity of suspensions. J. Fluid Mech. 554, 109-123.

Chen, S. \& Doolen, G. D. 1998 Lattice-Boltzmann method for fluid flows. Annu. Rev. Fluid Mech. 30, 329-364.

Cichocki, B. \& Jones, R. B. 1998 Image representation of a spherical particle near a hard wall. Physica A 258, 273-302.

Derksen, J. \& Van Den AkKer, H. E. A. 1999 Large-eddy simulations on the flow driven by a Rushton turbine. AIChE J. 45, 209-221.

Didwania, A. K. \& Homsy, G. M. 1981 Flow regime and flow transitions in liquid-fluidized beds. Intl J. Multiphase Flow 7, 563-580.

Duru, P. \& GuAzelli, E. 2002 Experimental investigations on the secondary instability of liquidfluidized beds and the formation of bubbles. J. Fluid Mech. 470, 359-382.

Duru, P., Nicolas, M., Hinch, J. \& Guazelli, E. 2002 Constitutive laws in liquid-fluidized beds. J. Fluid Mech. 452, 371-404.

Eggels, J. G. M. \& Somers, J. A. 1995 Numerical simulation of free convective flow using the lattice-Boltzmann scheme. Intl J. Heat Fluid Flow 16, 357-364.

El-KaIssy, M. M. \& Homsy, G. M. 1976 Instability waves and the origin of bubbles fluidized beds. Part 1: Experiments. Intl J. Multiphase Flow 2, 379-395.

GidasPow, D. 1994 Multiphase Flow and Fluidization. Academic Press, CA.

Glasser, B. J., Kevrekidis, I. G. \& Sundaresan, S. 1996 One- and two-dimensional travelling wave solutions in gas-fluidized beds. J. Fluid Mech. 306, 183-221.

Glasser, B. J., Kevrekidis, I. G. \& Sundaresan, S. 1997 Fully developed travelling wave solutions and bubble formation in fluidized beds. J. Fluid Mech. 334, 157-188.

Goldstein, D., Handler, R. \& Sirovich, L. 1993 Modeling a no-slip flow boundary with an external force field. J. Comput. Phys. 105, 354-366.

Gondret, P., Lance, M. \& Petit, L. 2002 Bouncing motion of spherical particles in fluids. Phys. Fluids 14, 643-652.

Griffith, B. E. \& Peskin, C. S. 2005 On the order of accuracy of the immersed boundary method: higher order convergence rates for sufficiently smooth problems. J. Comput. Phys. 208, 75-105.

Ham, J. M., Thomas, S., Guazzelli, E., Homsy, G. M. \& Anselmet, M. C. 1990 An experimental study of the stability of liquid-fluidized beds. Intl J. Multiphase Flow 16,171-185.

Hill, R. J., Koch, D. L. \& LADD, A. J. C. 2001 Moderate-Reynolds-number flows in ordered and random arrays of spheres. J. Fluid Mech. 448, 243-278.

van der Hoef, M. A., Beetstra, R. \& Kuipers, J. A. M. 2005 Lattice-Boltzmann simulations of low-Reynolds-number flow past mono- and bidisperse arrays of spheres: results for the permeability and drag force. J. Fluid Mech. 528, 233-254.

JACKSON, R. 2000 Dynamics of Fluidized particles. Cambridge University Press.

Joseph, G. G., Zenit, R., Hunt, M. L. \& Rosenwinkel, A. M. 2001 Particle-wall collisions in a viscous fluid. J. Fluid Mech. 433, 329-346.

Kandhai, D., Derksen, J. J. \& Van Den Akker, H. E. A. 2003 Interphase drag coefficients in gas-solid flows. AIChE J. 49, 1060-1065.

Kim, S. \& Karrila, S. J. 1991 Microhydrodynamics: Principles and Selected Applications. Butterworth-Heinemann.

Koch, D. L. \& Sangani, A. S. 1999 Particle pressure and marginal stability limits for a homogeneous monodisperse gas fluidized bed: kinetic theory and numerical simulations. J. Fluid Mech. 400, 229-263.

LADD, A. J. C. 1994a Numerical simulations of particle suspensions via a discretized Boltzmann equation. Part 1. Theoretical Foundation. J. Fluid Mech. 271, 285-309.

LADD, A. J. C. $1994 b$ Numerical simulations of particle suspensions via a discretized Boltzmann equation. Part 2. Numerical results. J. Fluid Mech. 271, 311-339.

LADD, A. J. C. 1997 Sedimentation of homogeneous suspensions of non-Brownian spheres. Phys. Fluids 9, 491-499. 
Li, J. \& Kuipers, J. A. M. 2003 Gas-particle interactions in dense gas-fluidized beds. Chem. Engng Sci. 58, 711-718.

Lovalenti, P. M. \& Brady, J. F. 1993 The hydrodynamic force on a rigid particle undergoing arbitrary time-dependent motion at small Reynolds number. J. Fluid Mech. 256, 561-605.

Lun, C. K. K., Savage, S. B., Jeffrey, D. J. \& Chepurniy, N. 1984 Kinetic theories for granular flow - inelastic particles in Couette flow and slightly inelastic particles in a general flow field. J. Fluid Mech. 140, 223-256.

MAXEY, M. R. \& Riley, J. J. 1983 Equation of motion for a small rigid sphere in a nonuniform flow. Phys. Fluids 26, 883-889.

NGuYEn, N.-Q. \& LADD, A. J. C. 2002 Lubrication corrections for lattice-Boltzmann simulations of particle suspensions. Phys. Rev. E 66, 046708.

Pan, T. W., Joseph, D. D., BaI, R., Glowinski, R. \& Sarin, V. 2002 Fluidization of 1204 spheres: simulation and experiment. J. Fluid Mech. 451, 169-191.

Poletto, M., BaI, R. \& Joseph, D. D. 1995 Propagation of voidage waves in a two-dimensional liquid-fluidized bed. Intl J. Multiphase Flow 21, 223-239.

Potapov, A. V., Hunt, M. L. \& Campbell, C. S. 2001 Liquid-solid flows using smoothed particle hydrodynamics and the discrete element method. Powder Technol. 116, 204-213.

Qian, Y. H., D’Humieres, D. \& Lallemand, P. 1992 Lattice BGK for the Navier-Stokes equations. Europhys. Lett. 17, 479-484.

Richardson, J. F. \& ZAKi, W. N. 1954 Sedimentation and fluidization. Part 1. Trans. Inst. Chem. Engng 32, 35-53.

Schiller, L. \& NaUmanN, A. 1933 Uber die grundlagenden Berechnungen bei der Schwerkraftaufbereitung. Ver. Deut. Ing. Z. 77, 318-320.

Singh, P. \& JoSEPH, D. D. 1995 Dynamics of fluidized suspensions of spheres of finite size. Intl J. Multiphase Flow 21, 1-26.

Singh, P., Hesla, T. I. \& JoSEPH, D. D. 2003 Distributed Lagrange multiplier method for particulate flows with collisions. Intl J. Multiphase Flow 29, 495-509.

SOMERS, J. A. 1993 Direct simulation of fluid flow with cellular automata and the lattice-Boltzmann equation. Appl. Sci. Res. 51, 127-133.

Sundaresan, S. 2003 Instabilities in fluidized beds. Annu. Rev. Fluid Mech. 35, 63-88.

Ten Cate, A. \& Sundaresan, S. $2006 a$ Analysis of flow in inhomogeneous particle beds using the spatially averaged two-fluid equations. Intl J. Multiphase Flow 36, 106-131.

Ten Cate, A. \& Sundaresan, S. $2006 b$ Analysis of unsteady forces in ordered arrays. J. Fluid Mech. 552, 257-287.

Ten Cate, A., Nieuwstad, C. H., Derksen, J. J. \& Van den Akker, H. E. A. 2002 PIV experiments and lattice-Boltzmann simulations on a single sphere settling under gravity. Phys. Fluids 14, 4012-4025.

Ten Cate, A., Derksen, J. J., Portela, L. M. \& Van den Akker, H. E. A. 2004 Fully resolved simulations of colliding spheres in forced isotropic turbulence. J. Fluid Mech. 519, 233-271.

Torquato, S., Truskett, T. M. \& Debenedetti, P. G. 2000 Is random close packing of spheres well defined? Phys. Rev. Lett. 84, 2064-2067.

Verberg, R. \& КосH, D. L. 2006 Rheology of particle suspensions with low to moderate fluid inertia at finite particle inertia. Phys. Fluids 18, 083303-1-16.

WANG, J. W. \& GE, W. 2005 Collisional particle phase pressure in particle-fluid flows at high particle inertia. Phys. Fluids 17,128103-1-3.

Wylie, J. J., Koch, D. L. \& LADD, A. J. C. 2003 Rheology of suspensions with high particle inertia and moderate fluid inertia. J. Fluid Mech. 480, 95-118.

Yamamoto, Y., Ротthoff, M., Tanaka, T., Kajishima, T. \& Tsuji, Y. 2001 Large-eddy simulation of turbulent gas-particle flow in a vertical channel: effect of considering inter-particle collisions. J. Fluid Mech. 442, 303-334.

YAng, F.-L. \& Hunt, M. L. 2006 Dynamics of particle-particle collisions in a viscous liquid. Phys. Fluids 18, 121506-1-11.

Zenit, R. \& Hunt, M. L. 2000 Solid fraction fluctuations in solid-liquid flows. Intl J. Multiphase Flow 26, 763-781.

Zenit, R., Hunt, M. \& Brennen, C. E. 1997 Collisional particle pressure measurements in solidliquid flows. J. Fluid Mech. 353, 261-283. 